\title{
A Multiple Ideal Points: Revealed Preferences in Different Domains
}

\section{Scott Moser ${ }^{\oplus 1}$, Abel Rodríguez ${ }^{\oplus 2}$ and Chelsea L. Lofland ${ }^{3}$}

\author{
${ }^{1}$ Associate Professor, School of Politics and International Relations, University of Nottingham, Nottingham, UK. \\ scott.moser@nottingham.ac.uk,URL: http://www.nottingham.ac.uk/ /dzsm2/ \\ ${ }^{2}$ Professor, Department of Statistics, University of California, Santa Cruz, 1156 High Street, Mailstop SOE2, Santa Cruz, \\ CA 95064, USA. abel@soe.ucsc.edu, URL: http://soe.ucsc.edu/ abel \\ ${ }^{3}$ Department of Statistics, University of California, Santa Cruz, 1156 High Street, Mailstop SOE2, Santa Cruz, \\ CA 95064, USA. clofland@soe.ucsc.edu, URL: https://ams.soe.ucsc.edu/people/clofland
}

\begin{abstract}
We extend classical ideal point estimation to allow voters to have different preferences when voting in different domains-for example, when voting on agricultural policy than when voting on defense policy. Our scaling procedure results in estimated ideal points on a common scale. As a result, we are able to directly compare a member's revealed preferences across different domains of voting (different sets of motions) to assess if, for example, a member votes more conservatively on agriculture motions than on defense. In doing so, we are able to assess the extent to which voting behavior of an individual voter is consistent with a uni-dimensional spatial model-if a member has the same preferences in all domains. The key novelty is to estimate rather than assume the identity of "stayers"-voters whose revealed preference is constant across votes. Our approach offers methodology for investigating the relationship between the basic space and issue space in legislative voting (Poole 2007). There are several methodological advantages to our approach. First, our model allows for testing sharp hypotheses. Second, the methodology developed can be understood as a kind of partial-pooling model for item response theory scaling, resulting in less uncertainty of estimates. Related, our estimation method provides a principled and unified approach to the issue of "granularity" (i.e., the level of aggregation) in the analysis of roll-call data (Crespin and Rohde 2010; Roberts et al. 2016). We illustrate the model by estimating U.S. House of Representatives members' revealed preferences in different policy domains, and identify several other potential applications of the model including: studying the relationship between committee and floor voting behavior; and investigating constituency influence and representation.
\end{abstract}

Keywords: ideal point estimation, dimensionality, non-parametric Bayesian methods, roll-calls, multiple comparisons

\section{Introduction}

Measuring the preferences of political actors is an important-and increasingly necessary-tool in the political scientist's tool kit. Indeed since at least the seminal work of Poole and Rosenthal (1985), a cottage industry has emerged attempting to measure the preferences-or "ideology"-of political actors. ${ }^{1}$ However, since preferences are never directly observed, inferring them empirically can be tricky. The goal is then to estimate an unobserved, latent trait of political actors (e.g., members of congress, justices, municipalities, constituencies) based on observed behavior (e.g., votes, political donations, text, etc.). The resulting revealed preferences are useful, among other reasons, for theory testing. From scholarship on legislative bargaining (Krehbiel 1998) to executive-legislative relations (Cameron 2000) to voting and representation (Jessee 2012),

Corresponding author Scott Moser Conference under the title "Comparing Revealed Preferences Across Multiple Types of Motions in the 83rd to 112th, U.S. House of Representatives." This work has benefited from helpful comments from Marc Ratkovic, as well as from three anonymous referees. Results presented here can be reproduced at https://codeocean.com/capsule/5298256/.

1 The estimated latent traits and position thereon are often interpreted as ideology, for example, liberal-conservative in a one-dimensional setting (Jessee 2012). We, however, interpret the position on latent traits as revealed preferences of political actors, as these traits are estimated only on the basis of observed (i.e., revealed) behavior. 
preferences of political actors (median member of a legislative body; president and pivotal members of Congress; constituents and representatives, respectively) play a crucial role in theories of various political phenomena. Indeed, Clarke and Primo (2012) suggest such theory-testing is a characteristic of political science. ${ }^{2}$

By far the most dominant methodological and theoretical tool in modern political science for operationalizing the process of inferring preferences from data is the so called spatial model (Stokes 1963; Davis, Hinich, and Ordeshook 1970; Enelow and Hinich 1984), in which political actors' preferences are represented in Euclidean space. In this context, testing theories of political behavior and/or institutions often requires measuring (or estimating) the preferences of different groups of political actors (e.g., members of the U.S. House and members of the U.S. Senate) on a common scale. For example, veto bargaining in the domain of legislative-executive relations (McCarty and Poole 1995; Tsebelis 1995) requires the executive's and the legislature's preferences be measured on a common scale. ${ }^{3}$

The main issue in this line of work is how to ensure that the preferences of these different groups of political actors are indeed measured on a common scale. While each group of actors can be scaled independently using, for example, NOMINATE (Poole and Rosenthal 1991, 2011) or IDEAL (Clinton, Jackman, and Rivers 2004), ensuring that the resulting scales are comparable is a delicate matter. Common approaches to ensure comparable scales include: assuming some common actors' preferences do not change (Shor, Berry, and McCarty 2010; Treier 2011); using vignettes to anchor the scale (Bakker et al. 2014; Asmussen and Jo 2016); assuming some actors' preferences change in a highly parametric fashion (Martin and Quinn 2002); and/or ignoring the issue of scaling actors on a common scale and instead focus on aspects of the distribution of estimated ideal points invariant to certain transformations (Esteban and Ray 1994; Duclos, Esteban, and Ray 2004; Jessee and Theriault 2014). A less satisfying approach some authors take is to ignore the issue of comparability and treat scales based on different votes as equivalent (Binder 1999). Lofland, Rodríguez, and Moser (2017) discuss the limitations and disadvantages of such approaches.

Another version of this estimation problem-measuring the preferences of actors across different domains of voting-is less studied and there are fewer methods for performing such comparisons. Being able to make comparisons of a member's revealed preference in different domains is growing increasing useful for the study of legislatures. For example, in studying majority-minority party relations in the U.S. House, Egar (2016) compares voting behavior between two types of roll-calls: those requested by majority-party members and those requested by minority-party members. Theriault (2008) and Jessee and Theriault (2014) argue that partisan polarization in the U.S. House of Representatives is the result of differential party influence on member's voting behavior between procedural votes and votes on final passage. In these examples the main methodological challenge is to measure (or estimate) the preferences of a common set of political actors based on behavior in different domains (e.g., roll-call voting on procedural motions vs. roll-call voting on final passage motions) on a common scale. When scholarly inquiry requires this type of comparison-for example, between voting in the Committee of the Whole versus the House, as in Roberts and Smith (2003); between different issue areas, as in Jochim and Jones (2013); or between lame-duck sessions and nonlame-duck sessions, as in Nokken (2014)-votes are usually segregated by type and analyzed separately. A major disadvantage to this approach is the lack of comparability across scales (if one attempts to use the inferred ideal points for the comparison), and the challenges of comparing the rank orders (which are identifiable but difficult to compare, as we illustrate in Section 5.4).

2 See also Krehbiel, Meirowitz, and Woon (2005)

3 We say "the legislature" for simplicity. Many legislatures are bicameral with the different chambers playing different roles. In such cases each chamber's preferences-or more precisely the preferences of the relevant veto players in chamber (e.g., the median voter)-need be measured on a comparable scale. 
We develop a novel technique to estimate legislators' revealed preferences in different domains of voting on a common scale. In our approach, votes are broken into predetermined groups and legislators are, in principle, allowed to have different preferences for each group. A hierarchical Bayesian model that uses clustering priors is then used to shrink the number of distinct positions that a legislators might have. When all votes belong to the same group, or all legislators have identical positions in all issues, our model reduces to a standard Bayesian item response theory (IRT) model (Albert and Chib 1993; Clinton et al. 2004). Otherwise, our approach is a strict generalization of it that relaxes the usual assumption that all votes are "equal" (for purposes of estimating latent traits from roll-call votes). To ensure that the latent scales are comparable, our approach identifies individual whose ideal points do not change across voting domains. The approach is similar to the bridging-voters approach introduced in Shor et al. (2010) and Asmussen and Jo (2016) to link measurement scales across the federal and state legislatures in the United States. However, unlike Shor et al. (2010), our approach does not assume prior knowledge of the identity of the voters whose ideal points are identical across voting domains, and instead estimates it. ${ }^{4}$ This paper builds on the work of Lofland et al. (2017), in which a statistical model is developed to compare ideal points of legislators before and after an event, for example, the change in majority party following Senator Jeffords's (VT) party switch in the 107th U.S. Senate. Their model permits comparison of revealed preferences between two groups of votes. Here we extend this model to allow for an arbitrary number of groups by introducing a prior on the set of possible partitions of the groups inspired by the widely-used Chinese restaurant process prior (Pitman 1996).

In addition to avoiding ex ante assumptions about allowable changes in revealed preferences, our approach has several other methodological advantages. First, our estimation approach results in a common scale, which allows us to properly compare the preferences of individuals across voting domains. In particular, our approach allows for the direct comparison and formal hypothesis tests across domains. For example, we may now formally test claims of the form "preferences in domain $A$ are different than those in domain $B$ " at the individual and group level. Second, unlike most other approaches in the literature, ours allows us to make statements about the consistency of preferences at the individual-level, the group-level and at the chamber level. This is a direct consequence of the fact that our model estimates the identify of members with consistent preferences in all domains. Third, because it relies on a joint model across all voting domains, the methodology developed here is a kind of "partial pooling" model for roll-call votes/IRT scaling, resulting in more precise estimates of preferences in each voting domain (a feature analogous to the "information borrowing" found in multilevel models). Finally, since our model uses a hierarchical Bayesian prior, it automatically adjusts for the large number of comparisons involved in the analysis (Scott and Berger 2006, 2010).

We illustrate our model through an analysis of preferences in different policy domains in the U.S. House of Representatives using the taxonomy of votes defined by Policy Agendas Project (hereafter, PAP) major topic groups (Baumgartner and Jones 1993; Policy Agendas Project 2017). As discussed in Section 3, in this setting, the resulting multiple ideal points estimated by our model may be interpreted as issue-specific preferences. We are not the first to examine issue-specific preferences. Gerrish and Blei (2010, 2012a, 2012b) and Lauderdale and Clark (2014) use meta-data associated with a vote (specifically, the text of the motion or case being decided) to infer different aspects of voters' utility. Their approach is based on the idea that members have preferences over different issues and that votes are "about" issues in some proportion. They use the text of a motion to estimate what each vote is "about" (the issues present in a given vote). Despite the similarities in the methodological objectives of these works, there are several differences between

4 While there are some connections between our model and the bridging literature, the inferential goals and data constraints are different. 
Gerrish and Blei (2012b) and Lauderdale and Clark (2014), including identification, estimation, and the structure of the model. For example, in Gerrish and Blei (2012b) voters have a main ideal point and issue-specific adjustments there-from. Identification is met by the use of continuous shrinkage priors on deviations. In Lauderdale and Clark (2014), voters are allowed to have arbitrary preferences over each issue and identification is obtained by requiring each voter to weigh the issue-content of the vote the same. Our approach does not directly use the content of the bill. Instead we use manually curated groups of votes as our metadata (see Section 2 for details). 5

The rest of the paper is organized as follows. In Section 2, we present the Bayesian model and prior specification. Section 3 discusses the interpretation of the model, and the relationship between vote group clusters and the classical literature on dimensionality. Section 4 discusses estimation and identification procedures. Section 5 illustrates the model by examining the revealed preferences of House members in different policy issue areas, grouping roll-called votes by PAP major topic. Section 6 discusses the implications of our work and potential future applications of the methodology.

\section{Model}

Let $y_{i, j}=1$ if the vote cast by legislator $i=1, \ldots, I$ on motion $j=1, \ldots, J$ is "Yea" and $y_{i, j}=0$ if it is "Nay." Spatial voting models assume that legislators make decisions according to random utility functions that depend on the distance between the legislator's preferred policy (their ideal point) and that of a particular piece of legislation in an unobservable policy space. The exact form of the utility (e.g., quadratic as in Jackman 2001; Clinton et al. 2004 or Gaussian as in Poole and Rosenthal 1987; McCarty, Poole, and Rosenthal 1997) and the random shocks (e.g., Gaussian vs. extreme value) differ across models. ${ }^{6}$ In the case of a quadratic loss function with independent errors over preferences that lie in a $D$-dimensional Euclidean policy space, this leads to a likelihood of the form

$$
y_{i, j} \mid \mu_{j}, \boldsymbol{\alpha}_{j}, \boldsymbol{\beta}_{i} \sim \operatorname{Bernoulli}\left(y_{i, j} \mid G\left(\mu_{j}+\boldsymbol{\alpha}_{j}^{T} \boldsymbol{\beta}_{i}\right)\right),
$$

where $\boldsymbol{\beta}_{i} \in \mathbb{R}^{D}$ corresponds to the position of legislator $i$ in policy space (their ideal point), $\mu_{j}$ controls the baseline probability of a positive vote (Yea) on motion $j, \boldsymbol{\alpha}_{j} \in \mathbb{R}^{D}$ controls the effect of the ideal points of the legislators on the probability of a positive vote on motion $j$, and $G$ is an appropriate link function. ${ }^{7}$ For the purpose of this paper, we assume that $G$ is the cumulative distribution function of the standard normal distribution, that is, we work with a probit model.

We extend this spatial voting model to account for legislators with potentially different ideal points associated with each of $K$ mutually exclusive groups of motions. The groups of motions are identified through (known) indicator variables $\gamma_{1}, \ldots, \gamma_{J}$ such that $\gamma_{j} \in\{1, \ldots, K\}$, that is, $\gamma_{j}=k$ if and only if motion $j$ is in group $k$. For example, in the illustration discussed in Section $5, \gamma_{j}$ indicates to which one of the major PAP categories a vote belongs to. ${ }^{8}$ The likelihood of this extended model

5 There are additional technical differences between our work and theirs. The Gerrish and Blei (2012b) model does not allow for testing sharp hypotheses directly (you have to postprocess the estimates in a more or less ad hoc way), where as we can. Further, our approach results in legislator-specific clustering of domains not possible in Lauderdale and Clark (2014).

6 See Carroll et al. (2009) and Clinton and Jackman (2011) for more on the differences between variations on random utility models and see Carroll et al. (2013) for an empirical comparison of different functional forms

7 This familiar two-parameter IRT model (Lord 1952; Rasch 1960) formulation can be derived from a more micro-level account of voting behavior. For example, the usual quadratic-normal voting model can be described as follows. Each motion $j$ corresponds to a policy position if passed, $Y_{j}$ and a status quo policy if it fails, $N_{j}$, each a point in $\mathbb{R}^{D}$. Voter $i$ gets utility $U_{i}\left(Y_{j}\right)=-\left\|Y_{j}-\boldsymbol{\beta}_{i}\right\|^{2}+\eta_{i j}$ if motion $j$ passes and utility $U_{i}\left(N_{j}\right)=-\left\|N_{j}-\boldsymbol{\beta}_{i}\right\|^{2}+v_{i j}$ if it fails, where $\|\cdot\|$ is the Euclidean norm. If we assume the errors are jointly normal with $E\left(\eta_{i j}\right)=E\left(v_{i j}\right), V \operatorname{ar}\left(\eta_{i j}-v_{i j}\right)=\sigma_{j}^{2}$ and with $\eta$ and $\mu$ independent across voters and motions, then simple algebra shows $\alpha_{j}=2\left(Y_{j}-N_{j}\right) / \sigma_{j}$ and $\mu_{j}=\left(Y_{j}^{\prime} Y_{j}-N_{j}^{\prime} N_{j}\right) / \sigma_{j}$ and $G$ becomes the cumulative normal distribution function in (1). See Londregan (1999) and Clinton and Jackman (2011) for more on the connections between ideal point estimation from binary voting data and IRT.

8 One may question our assumption that vote-types are nominal (in the statistics literature this difference is analogous to the different between block models and mixed-membership models). For example, Gerrish and Blei (2012b) and Lauderdale 
takes the form

$$
y_{i, j} \mid \mu_{j}, \boldsymbol{\alpha}_{j}, \boldsymbol{\beta}_{i, k}, \gamma_{j} \sim \operatorname{Bernoulli}\left(y_{i, j} \mid G\left(\mu_{j}+\boldsymbol{\alpha}_{j}^{T} \boldsymbol{\beta}_{i, \gamma_{j}}\right)\right),
$$

where $\boldsymbol{\beta}_{i, 1}, \ldots, \boldsymbol{\beta}_{i, K}$ correspond to the (potentially distinct) ideal points of legislator $i$ on each of the groups of motions. Hence, when $K=1$, our model reduces to the usual IRT scaling of votes (e.g., Clinton et al. 2004) .

Similar to Lofland et al. (2017), we introduce a joint prior for $\boldsymbol{\beta}_{i, 1}, \ldots, \boldsymbol{\beta}_{i, K}$ that allows us to identify groups of motions for which a given legislator has the same revealed preferences. In this case, the prior is inspired by ideas from model-based clustering. More specifically, we rewrite the ideal point of legislator $i$ for group $k$ as $\boldsymbol{\beta}_{i, k}=\tilde{\boldsymbol{\beta}}_{i, \zeta_{i, k}}$, where $\left\{\tilde{\boldsymbol{\beta}}_{i, 1}, \tilde{\boldsymbol{\beta}}_{i, 2}, \ldots\right\}$ represent the set of unique ideal points possessed by individual $i$, and the (unknown) auxiliary variables $\zeta_{1}, \ldots, \zeta_{I} \in\{1,2, \ldots\}$ are such that $\zeta_{i, k}=\zeta_{i, k^{\prime}}$ if and only if legislator $i$ possesses the same ideal point in the $k$ th and the $k^{\prime}$ th groups of motions. Hence, the indicators $\zeta_{i, 1}, \ldots, \zeta_{i, K}$ partition the set $\left\{\boldsymbol{\beta}_{i, 1}, \ldots, \boldsymbol{\beta}_{i, K}\right\}$ into $L_{i} \leq K$ legislator-specific clusters; note that if $\zeta_{i, k}=\zeta_{i, k^{\prime}}$, then legislator $i$ has the same preferences over votes in group $k$ as for votes in group $k^{\prime}$. This structure resembles that of a collection of $I$ independent finite mixture models, each one associated with a different legislator. We call legislators for which $\zeta_{i, k} \neq \zeta_{i, k^{\prime}}$ movers between vote group $k$ and vote group $k^{\prime}$, and those for which $\zeta_{i, k}=\zeta_{i, k^{\prime}}$ stayers. Similarly, if $\zeta_{i, k}=\zeta_{i, k^{\prime}}$ for all $k$ and $k^{\prime}$, then legislator $i$ exhibits the same preferences on all votes and we call them a full stayer. If all legislators are full stayers, the extended model also reduces to the one in (1).

As mentioned in the introduction, while the approach of Shor et al. (2010) and Shor and McCarty (2011) assumes the vectors $\boldsymbol{\zeta}_{1}, \ldots, \boldsymbol{\zeta}_{I}$ are known in advance, we instead treat these indicators as unknown parameters and aim to estimate them from the data. This requires that we specify a (joint) prior distribution for the indicator vectors. Following Gopalan and Berry (1998), it would be natural to assign independent Chinese restaurant priors (Antoniak 1974) with a common dispersion parameter $\phi>0$ to each vector $\zeta_{i}$, that is,

$$
p\left(\zeta_{1}, \ldots, \zeta_{I} \mid \phi\right)=\prod_{i=1}^{I}\left\{\frac{\Gamma(\phi)}{\Gamma(\phi+K)} \phi^{L\left(\zeta_{i}\right)} \prod_{I=1}^{L\left(\zeta_{i}\right)} \Gamma\left(n_{l}\left(\zeta_{i}\right)\right)\right\},
$$

where $\Gamma(t):=\int_{0}^{\infty} z^{t-1} \exp \{-z\}$ is the well-known Gamma function, $L\left(\zeta_{i}\right)=L_{i}$ is the number of unique values among $\zeta_{i, 1}, \ldots, \zeta_{i, K}$ (i.e., the number of clusters for legislator $\left.i\right),{ }^{9} 1(\cdot)$ is the indicator function, and $n_{l}\left(\zeta_{i}\right)=\sum_{k=1}^{K} 1\left(\zeta_{i, k}=l\right)$ is the number of groups of motions assigned to cluster $l$. Under this model, the prior probability that a given legislator is a stayer between two groups of votes is $\frac{1}{1+\phi}$, the probability that a given legislator is a full stayer is $\theta:=\frac{\Gamma(K) \Gamma(1+\phi)}{\Gamma(K+\phi)}$, and the prior number of full stayers, $B$, follows a binomial distribution with size $I$ and probability $\theta$.

While (3) has a number of appealing features, the use of fully independent priors for each legislator does not ensure that the different latent spaces share a common scale. Indeed, as discussed in Lofland et al. (2017), a minimum number of stayers (dependent on the dimension $D$ of the latent space) are required to ensure identifiability across mutually exclusive groups of votes. A sufficient condition that is also easy to enforce computationally is the presence of at least $D+1$ full stayers. Hence, in this paper we work with the modified prior

and Clark (2014) allow votes to be "about" a mixture of topics. While both approaches have merit and are applicable to related research questions, we argue that treating vote-type as categorical is naturally appropriate in some settings: votes adopting restrictive rules are categorically different than other types of procedural votes (Roberts 2010; Moffett 2012); voting on a motion to recommit is categorically different from, for example, voting on final passage (Duff and Rohde 2012).

9 We discuss the conceptual connections between clusters and dimensions in Section 3. In the illustration, we argue that the quantity $L_{i}$ can be interpreted as the dimensionality of the preferences of legislator $i$. 


$$
p\left(\zeta_{1}, \ldots, \zeta_{I} \mid \phi\right)=\frac{\prod_{i=1}^{I}\left\{\frac{\Gamma(\phi)}{\Gamma(\phi+K)} \phi^{L\left(\zeta_{i}\right)} \prod_{l=1}^{L\left(\zeta_{i}\right)} \Gamma\left(n_{l}\left(\zeta_{i}\right)\right)\right\} 1\left(\zeta_{1}, \ldots, \zeta_{I} \in \Omega\right)}{1-\sum_{s=0}^{D+1}\left(\begin{array}{l}
I \\
s
\end{array}\right)\left(\frac{\Gamma(K) \Gamma(1+\phi)}{\Gamma(K+\phi)}\right)^{s}\left(1-\frac{\Gamma(K) \Gamma(1+\phi)}{\Gamma(K+\phi)}\right)^{I-s}},
$$

where $\Omega$ is the set of possible $\left\{\boldsymbol{\zeta}_{1}, \ldots, \boldsymbol{\zeta}_{I}\right\}$ containing at least $D+1$ full stayers. The normalizing constant in (4) corresponds to the probability of the set $\Omega$ under the unrestricted model.

Note that the hyperparameter $\phi$ plays a key role in controlling the number of stayers. Hence, rather than fixing it in advance, we attempt to learn it from the data by assigning it a hyperprior in such a way that the implied prior on $\theta$, the probability that any one legislator is a full stayer, is uniform (see Supplementary Material A for a closed-form expression for this prior). This choice is appealing for a variety of reasons. First, making $\theta$ random ensures that the model automatically adjusts for multiplicities (Scott and Berger 2006, 2010). This addresses concerns related to the large numbers of comparisons that are intrinsically being made by our model. Second, a uniform prior on $\phi$ implies a uniform prior on the number of stayers for all values of $K$, which avoids biases that might arise from the fact that there are many more configurations with around $I / 2$ stayers than there are configurations with either a very small or a very large number of them. ${ }^{10}$ Finally, under a uniform prior for $\theta$, and for the special case $K=2$, our model matches the one developed in Lofland et al. (2017). We assessed the sensitivity of the model to this prior choice by refitting the model using two alternative Gamma priors on $\phi$. First, we considered a Gamma prior with shape parameter 5 and scale 1, which implies a prior on $\theta$ that has mean 0.002 and $95 \%$ prior credible interval of $(0,0.014)$. Next, we considered a Gamma prior with shape parameter 0.075 and scale parameter 1 , which implies a prior on $\theta$ with mean 0.90 and $95 \%$ prior credible interval $(0.10,1.00)$. The results were the same under all three priors.

In addition to priors on the indicator variables $\boldsymbol{\zeta}_{1}, \ldots, \boldsymbol{\zeta}_{I}$, we need to specify priors for each component of the unique ideal points $\tilde{\beta}_{i, k, d}, i \in\{1, \ldots, I\}, k \in\{1, \ldots, K\}, d \in\{1, \ldots, D\}$. As in Lofland et al. (2017), we assign these parameters independent Gaussian priors with common location and scale parameters, $\tilde{\beta}_{i, k, d} \mid \eta_{d}, \sigma_{d}^{2} \sim \mathrm{N}\left(\eta_{d}, \sigma_{d}^{2}\right)$, where the hyperparameters $\eta_{d}$ and $\sigma_{d}^{2}$ are given independent, conditionally conjugate priors, $\eta_{d} \sim \mathrm{N}(0,1)$ and $\sigma_{d}^{2} \sim \operatorname{IGam}(2,1)$ for every $d \in\{1, \ldots, D\}$. In terms of the bill-specific parameters, we follow the literature and work with conditionally conjugate priors, $\mu_{j} \mid \rho, \kappa^{2} \sim \mathrm{N}\left(\rho, \kappa^{2}\right)$ and $\alpha_{j, d} \mid \omega_{d}, \tau^{2} \sim \omega_{d} \delta_{0}+\left(1-\omega_{d}\right) \mathrm{N}\left(0, \tau^{2}\right)$ independently for all votes $j$ and dimensions $d$, and where $\delta_{0}(\cdot)$ denotes the degenerate measure placing probability one at zero. The use of a zero-inflated prior for $\alpha_{j, d}$ enables us to automatically discount the effect of unanimous bills without explicitly dropping them from the analysis. Finally, the hyperparameters $\rho, \kappa^{2}, \omega_{d}$, and $\tau^{2}$ are given priors $\rho \sim \mathrm{N}(0,1), \kappa^{2} \sim \operatorname{IGam}(2,1)$, $\omega_{d} \sim \operatorname{Beta}(1,1 / d)$ and $\tau^{2} \sim \operatorname{IGam}(2,1)$. Besides being in line with priors that are widely used in the literature, our experience is that the model is also quite robust to the choice of these hyperparameters.

Ensuring that the scales associated with the different vote types, as the formulation introduced above does, is a nontrivial problem. Simply fitting independent models to each vote type would only allow us to compare identifiable quantities, such as the rank order of the legislators. However, as we have argued in the introduction, and illustrate in Section 5.4, comparing ranks can be very misleading, especially when the number of votes in each vote type is relatively small. One alternative approach a practitioner may be tempted to employ to ensure comparability is to use the same set of voters to anchor the ends of the scale for each vote group. For example, constraining party whips to be the extreme voters in each issue area. However, in such a strategy,

10 These issues appears in many other settings, including variable selection in linear models Scott and Berger (2010), and in estimation of conditional independence relationships in Gaussian graphical models (Armstrong et al. 2009). 
the identity of the set of full stayers is assumed, rather than estimated. Therefore, one loses the ability to test the hypotheses that, for example, the whips are indeed stayers. Our model retains that ability. Second, if $D>1$ and there are fewer than $D+1$ parties, then fixing two whips as anchors is not enough to get comparability. Lastly, while having two well-selected anchors when $D=1$ is enough for comparability of the scales, the question of how many full stayers there are in a given chamber is still important, both because (1) it leads to metrics like the $S F$ and ASF that are interesting by themselves (see Sections 4 and 5.3 below), and (2) it implicitly reduces the number of parameters that need to be estimated.

\subsection{Relationship with Standard Multidimensional Preference Models}

One may naturally wonder about the relationship between the number of vote types, $K$, and the dimension of the policy space, $D$. In this section we show our model is not a special case of a standard multidimensional IRT model (except in the trivial cases when all legislators are stayers or $K=1$ ). In particular, the specification in (2) with $D=1$ (which we use in our illustration) is not a special case of (1) with $D=K$. Instead we argue that it is better to think of our approach as one in which there is common space to measure preference in (even if the measurement is multidimensional). For example, a $K>1, D=2$ model places ideal points in two-dimensional space, but allows voters to have different (two-dimensional) ideal points in potentially $K$ different domains of voting.

Note that the interaction term $\boldsymbol{\alpha}_{j}^{T} \boldsymbol{\beta}_{i}$ in (1) has a simple bilinear structure in which one of the terms depends exclusively on the identity of the legislator, and the other depends exclusively on which measure is being voted on. This separable structure is a consequence of the assumption that the positions of legislators in the policy space are independent of the positions of the bills. This assumption underlies traditional spatial voting models, including IDEAL and NOMINATE. A further consequence of the independence assumption is that the matrix of factor loadings is the same for every legislator. That is, while each legislator has its own unique preferred policies, the weights associated with each of the dimensions on a given vote is the same for all legislators. Our model relaxes this assumption by allowing different loadings matrices for each legislator (see Equation 2).

A small example might be helpful in further clarifying the differences. Assume that there are only four measures to vote on (i.e., $J=4$ ), two for each of two vote types (i.e., $K=2$ ), and only two legislators (i.e., $I=2$ ). If the first legislator is a stayer but the second legislator has different ideal points for each vote type, the interaction terms associated with each of the four votes reduce to

$$
\left(\begin{array}{ll}
\alpha_{1} & 0 \\
\alpha_{2} & 0 \\
\alpha_{3} & 0 \\
\alpha_{4} & 0
\end{array}\right)\left(\begin{array}{l}
\beta_{1,1} \\
\beta_{1,2}
\end{array}\right) \quad \text { and } \quad\left(\begin{array}{cc}
\alpha_{1} & 0 \\
\alpha_{2} & 0 \\
0 & \alpha_{3} \\
0 & \alpha_{4}
\end{array}\right)\left(\begin{array}{l}
\beta_{2,1} \\
\beta_{2,2}
\end{array}\right)
$$

for the first and second legislators, respectively. By definition, standard IRT models cannot accommodate this type of structured interaction terms.

\section{Clustering and Dimensionality}

The model just introduced can be used to bear on the discussion of dimensionality of classical voting models, which has been a popular topic in the political science literature (see, e.g., Koford 1989; Wilcox and Clausen 1991; Potoski and Talbert 2000; Talbert and Potoski 2002; Aldrich, Montgomery, and Sparks 2014; Dougherty, Lynch, and Madonna 2014; Roberts, Smith, and Haptonstahl 2016). 
What constitutes a dimension is surprisingly not widely agreed upon. In a common interpretation, each dimension is associated with a set of substantive issues (in two-dimensional models, typically economic and social issues). This interpretation relies on the often held-but rarely checked-assumption that voting dimensions align with the content of the bills. ${ }^{11}$ However, at a technical level, the dimensionality of the voting is simply the number of latent traits required to accurately model legislators' voting behavior. Hence, from a technical point of view, choosing the dimensionality of the policy space is essentially a model selection problem in which we aim to balance model fit with model complexity, and the dimensions in the latent space of a spatial voting model do not need not correspond to any politically relevant substantive issues. Recognizing this, Poole (2007) makes the useful distinction between a basic space and an issue space. In this section, we build on this distinction to provide an interpretation for our model in situations in which the indicators $\gamma_{i, 1}, \ldots, \gamma_{i, J}$ partition the bills into groups that correspond to substantive issues for each legislator $i$ (such as in the illustration in Section 5).

Standard spatial voting models assume that every legislator has preferences over the same set of issues-that the dimension of the space is fixed and common. Motions load differently on each of these shared dimensions (however they are interpreted) to determine how a specific legislator votes. Therefore, the voting behavior of legislators for all motions can be explained as a linear combination of the latent features; bills ostensibly focused on substantive issues would be expected to have similar loadings on dimensions, and those loadings are the same for every legislator. In the specific case of a one-dimensional voting model, each vote can be explained by differentially weighting a single, legislator-specific latent trait. If the voting patterns for a substantial group of bills cannot be well explained in this way, then a two-dimensional voting model might be more appropriate. When the group of bills that is not well explained by the one-dimensional model happen to correspond to those bearing on a well-defined set of substantive issues, then the basic and issue spaces agree and the traditional interpretation of the dimensions of the latent space in terms of issues is appropriate. However, if the set of bills that require the additional dimension do not align with particular substantive issues, then the validity of this traditional interpretation of the dimensions is suspect and it becomes important to distinguish between the dimension of the basic space and the dimension of the issue space. Furthermore, in either case, if a dimension is added, it is added for all legislators even if this additional latent trait does not contribute to explaining the voting pattern of some voters. If that is the case, the corresponding ideal point on the added dimension will unnecessarily increase model complexity (via the number of parameters to be estimated for the legislators for which the additional dimension is irrelevant).

Our model works in a subtly different way. If linear combinations of a common set of lowdimensional latent traits are capable of explaining the voting behavior of most legislators for every substantive issue, our model will simply identify most of the legislators as full stayers. As discussed before, in that case our model corresponds to a traditional spatial voting model (as would be expected). However, if there is a subset of legislators whose voting pattern on certain groups of votes cannot be well explained through a common set of linear combinations of the latent traits, our model will introduce an additional set of legislator-specific ideal points. In the case in which the dimension of the basic space is $D=1$, and the bill groups being compared reflect substantive issues, the total number of ideal points associated with these particular individuals can be interpreted as the dimension of their preferences (i.e., the dimension of legislator-specific issue spaces). In this way, our model allows us to investigate the interrelation between substantive issues and the intrinsic dimensionality of the policy space.

Finally, we discuss our model in light of the interpretation of dimensions in spatial models. Benoit and Laver (2012) and De Vries and Marks (2012) discuss theoretical, epistemological, and

11 There are some notable exceptions, namely, Wilcox and Clausen (1991); Crespin and Rohde (2010); Lee and Schutte (2017). 
methodological issues when "dimensionalizing" political space. Broadly speaking, there are two approaches: a posteriori (inductive) and a priori. In the former dimensions are specified in advance of measurement/ estimation. Examples of such approaches include the policy-dimensions work of, for example, Clausen and Wilcox (1987) and Wilcox and Clausen (1991). In the latter, dimensions are estimated as latent traits, and interpreted ex post. The classical NOMINATE and related models are an example of this approach (Poole and Rosenthal 1985). In either approach, the key epistemological challenge is the same: "The 'spaces' of interest are ultimately metaphors and both the dimensions spanning these spaces and agents' positions on these dimensions are fundamentally unobservable." (Benoit and Laver 2012, p. 196) Our approach may the thought of as an intermediate compromise to the determination of "substantively relevant dimensions" in the language of Benoit and Laver (2012), having both inductive and deductive components. Namely, we start with (strong) priors on the structure of the conceptual space (by placing each vote in exactly one category we are effective putting a prior of $100 \%$ that a vote $v$ is of type $g$ ). And then inductively estimate the "... bundles of particular salient policy issues on which agents' preferences are inter-correlated” (Benoit and Laver 2012, p. 205). This interpretation requires the vote-categories to be (at least prima face) related to dimensions spanning the conceptual space.

\section{Estimation}

Estimation of the model parameters is performed using a Markov chain Monte Carlo (MCMC) algorithm (Robert and Casella 2005). To facilitate computation, we introduce auxiliary random variables

$$
z_{i, j} \mid \mu_{j}, \boldsymbol{\alpha}_{j}, \boldsymbol{\beta}_{i, k}, \gamma_{j} \sim \mathrm{N}\left(\mu_{j}+\boldsymbol{\alpha}_{j}^{T} \boldsymbol{\beta}_{i, \gamma_{j}}, 1\right),
$$

such that $y_{i, j}=1$ if $z_{i, j} \geq 0$ and $y_{i, j}=0$ otherwise (Albert and Chib 1993). Conditional on these auxiliary variables, the full conditional distributions for $\mu_{j}$ and $\alpha_{j, d}$ follow a normal and an inflated normal distribution, respectively. Similarly, the parameters $\rho, \kappa^{2}, \omega_{d}$, and $\tau^{2}$ follow Gaussian, inverse gamma, beta, and inverse gamma posterior distributions, respectively. On the other hand, the indicators $\zeta_{i, k}$ and the unique ideal points $\tilde{\boldsymbol{\beta}}_{i, k}$ are sampled using a slight variant of the collapsed Gibbs sampler described in Neal (2000). The associated concentration parameter $\phi$ is sampled using a random walk Metropolis Hastings on the logarithmic scale. Finally, the hyperparameters $\eta_{d}$ and $\sigma_{d}^{2}$ have Gaussian and Inverse Gamma full conditional posterior distributions, respectively. To mitigate well-known issues with potential multimodality in mixture models, we execute multiple runs of the MCMC algorithm starting at overdispersed points, and monitor the (unnormalized) posterior distribution of the model, as well as the ASF metric described below. Convergence for each of the chains is assessed using the criteria in Geweke (1992).

We use a number of summaries of posterior samples to address substantive questions of interest. One chamber-level summary we report is the average staying frequency,

$$
A S F=\frac{1}{I} \sum_{i=1}^{I} 1\left(\zeta_{i, 1}=\zeta_{i, 2}=\cdots=\zeta_{i, K}\right),
$$

that is, the proportion of full stayers. Values of the $A S F$ close to 1 indicate that most legislators exhibit a single set of revealed preferences across all vote types. In particular, if $A S F=1$ then our analysis of that particular set of votes is equivalent to fitting a $D$-dimensional IDEAL model to the data. Similarly, we also construct issue-specific $A S F$ metrics.

In addition to chamber-level measures of consistency we also construct micro-level metrics. These metrics involve the legislator-specific partitions of votes implied by the indicators $\zeta_{1}, \ldots, \zeta_{I}$. We construct point estimates for the partitions by minimizing an expected loss function based 
on pairwise clustering probabilities, $D_{k, k^{\prime}, i}=\operatorname{Pr}\left(\zeta_{i, k}=\zeta_{i, k^{\prime}} \mid\right.$ Data) (see Lau and Green 2007 for details). As we discussed before, in the context of the application we introduce in Section 5 , this partition provides information about the dimensionality of the issue space, and by extension, about how various substantive issues are correlated. Finally, we construct estimates of issuespecific legislators' preferences, $\boldsymbol{\beta}_{i, k}$ in order to systematically characterize differences in voting patterns, both at the issue and the legislator levels.

\subsection{Identifiability}

Two key identifiability issues need to be addressed in order to utilize the posterior samples from the algorithm. The first one relates to the well-known label switching problem that arises in mixture models (Celeux 1998; Stephens 2000). We avoid this problem by focusing our inference procedures on quantities that are invariant to the relabeling of the classes, such as $A S F, D_{k, k^{\prime}, i}$, or $\boldsymbol{\beta}_{i, k}$. The second one relates to the arbitrariness of the latent scale on which preferences are being embedded. Indeed, although the use of a minimum number of stayers in our prior on $\boldsymbol{\zeta}_{1}, \ldots, \boldsymbol{\zeta}_{I}$ ensures that the different groups of bills share a measurement scale, that common scale is still arbitrary. To resolve this issue we add a further constraint by fixing the location of the first component of the ideal point vector for $D+1$ legislators (Rivers 2003). We call these legislators anchors (as opposed to the stayers discussed above in the context of linking the different latent scales together). For example, in the unidimensional setting $(D=1)$ we might fix $\beta_{i_{1}, 1}=-1$ and $\beta_{i_{2}, 1}=1$, where $i_{1}$ and $i_{2}$ identify the whips of the two main parties in the legislature. These constraints are enforced by postprocessing the posterior samples, an approach sometimes referred to as parameter expansion (Liu, Rubin, and Wu 1998; Bafumi et al. 2005). The identity of the anchors has no effect on the posterior inferences associated with $\boldsymbol{\zeta}_{1}, \ldots, \boldsymbol{\zeta}_{I}$ and, in our experience, only a negligible impact on inferences for the ideal points themselves (Lofland et al. 2017).

\section{Illustration: Voting Patterns by Policy Issue}

We illustrate the model through an analysis of preferences in different policy domains using recorded votes from the 97th to 114th U.S. House of Representatives (1981-2016). Motions brought to a vote in the U.S. Congress differ thematically by substantive issue (e.g., the economy, environment, criminal justice, etc.). We use the PAP main topic coding to categorize roll call votes by issue area (Adler and Wilkerson 2017; Policy Agendas Project 2017). This taxonomy, which consists of 20 mutually exclusive major topics (e.g., Macroeconomics, Health, Environment, etc.), has been used to study legislative agendas (Baumgartner and Jones 1993) and attention (Jones and Baumgartner 2005), among other matters. ${ }^{12}$ We focus on the PAP categorization for two reasons. First, the application to issue areas illustrates the novel technical contributions of the model, namely (1) comparable revealed preferences across issues and (2) estimates of legislatorspecific dimensionality. Second, as argued in Section 3, applying the model to votes grouped by issue area is relevant to the literature on dimensionality (Talbert and Potoski 2002; Aldrich et al. 2014; Roberts et al. 2016) by providing issue-specific revealed preferences and an estimate of individual consistency-the posterior probably that a member has the same revealed preference in each issue area. However, we stress this is only an illustration of the model. We discuss possible additional applications to various questions in political science in Section 6.1.

We fit the model described in the previous sections independently to each of the 18 Houses mentioned above. We work with $D=1$, which is both a common choice in the U.S. House (Poole, Rosenthal, and Koford 1991; Poole 2007), and a convenient one in terms of model interpretation

12 This taxonomy, while popular and widely used, could be criticized on a number of points (Dowding, Hindmoor, and Martin $2013,2016)$. Most notably, one may wonder if a roll-called vote fits cleaning into one and only one topic. While such considerations are valid, they are not of concern in the present work. 


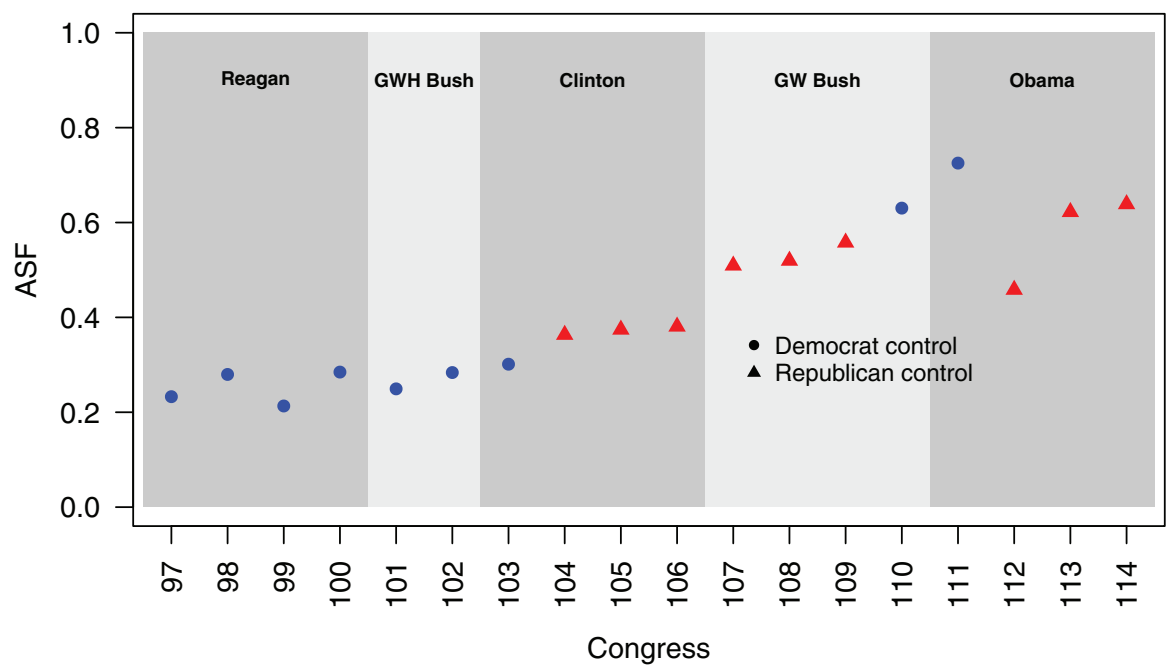

Figure 1. Average Staying Frequencies $(A S F)$ for the 97 th to 114 th U.S. Houses. The symbol and color of the points indicate the party on control of the chamber, while the grey area separate different presidencies.

(recall our discussion in Section 3). We start the analysis from the early 1980s as this is comfortably after the reforms of the mid-1970s. We end in the 114th due to data availability. Because of the rarity of some topics, we only include topics that tend to consistently have at least 20 votes in most Houses. The resulting data-set has $K=17$ groups. ${ }^{13}$ We have also removed from the dataset any legislator that missed more than $25 \%$ of the votes taken during the session. ${ }^{14}$

\subsection{Aggregate Analysis}

Figure 1 presents the posterior mean of the ASF defined in Equation (5), $E$ [ASF|Data]. The symbol and color of the points indicate the party on control of the chamber, while the grey area separate different presidencies. We can see that the staying frequency in the U.S. House of Representatives remained low and relatively stable during the Reagan and GWH Bush presidencies, but increased steadily after that. That is, until the early 1990s we find evidence of considerable differences in issue-specific preferences. The main exception to this pattern is the 112nd House, when ASF dropped sharply. The 112nd House corresponds to the second half of the first term of President Obama. Not only was this a House in which the Republican party retook the chamber, but it also saw an influx of new legislators that rode the Tea Party wave into office. The willingness of these legislators to vote against their party leadership in a number of issues might explain the relatively low value of the $A S F$ during this session of the House..$^{15}$

We can also disaggregate the $A S F$ results across PAP issues. Figure 2 shows posterior means for issue-specific staying frequencies. To construct these frequencies, we define the base cluster for each legislator as their cluster of issues that contains the most votes. For legislators that are full stayers, all issues belong to their base cluster. We define issue-specific average staying frequencies as the probability that an issue belongs to a member's respective base cluster, averaged over all

13 Specifically, we drop votes having PAP major code 9 (Immigration), 13 (Social Welfare), and 18 (Foreign Trade). We give the complete frequency of vote-types by Congress in Supplementary Material B. Eliminating these three topics does not reduce the total number of measures included in the analysis by much: the percentage of votes included in the analysis varies between $91 \%$ (for the 98th House) and $97 \%$ (for the 112th House), with a median of $95 \%$.

14 Although this threshold for inclusion might seem high, we still retain most of the legislators in every session: the number fluctuates between 402 (in the 97th House) and 428 (in the 109th and 113rd Houses), with a median of 424.

15 The rise in extra-dimensionality of voting in the 112th is curious and left for future study. An alternative explanation to the Tea Party voters might involve the agenda. We note that this session saw more Energy, Environment, and Defense motions and fewer recorded votes on International Affairs and Government Operations than the preceding sessions. Another possible explanation may lie in the interplay of vote type (e.g., amendment, final passage) and issue area. We note that Jochim and Jones (2013) find an increase in amendments in an issue-area corresponds with an increase in dimensionality of that issue (but that this relationship has diminished over time). 


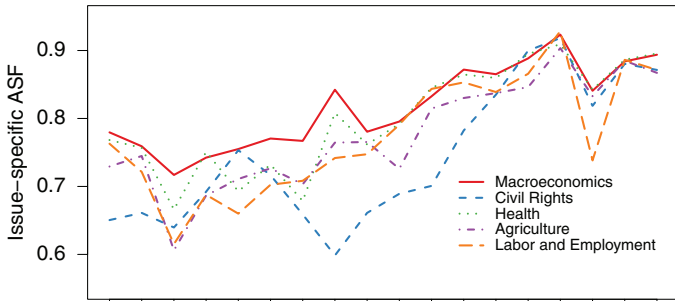

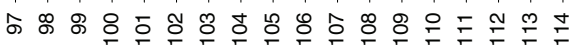

Congress

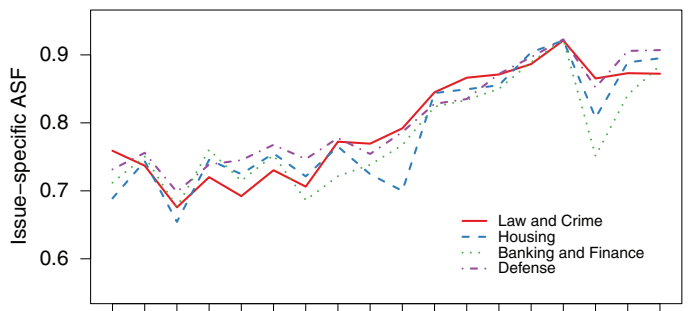

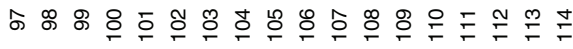

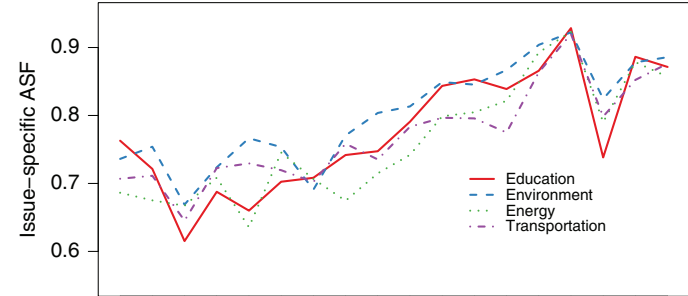

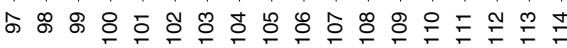
Congress

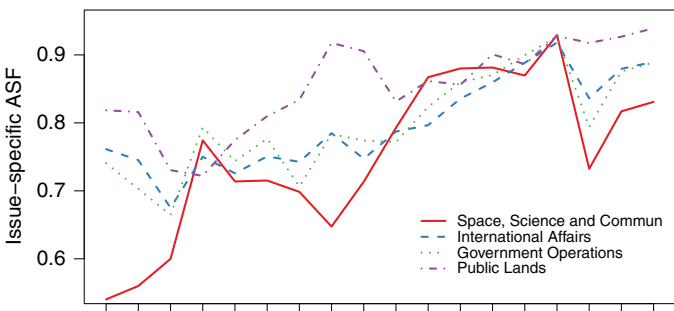

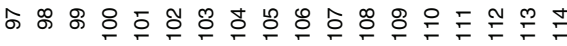
Congress

Figure 2. Probability of an issue being deviant (97th to 114th Houses).

legislators. These issue-specific ASFs can be interpreted roughly as the propensity for an issue to be "extra-dimensional." Formally it is the posterior probability that an issue is deviant-exhibits different preferences from a member's preferences in their base cluster. Overall, the pattern we observed for the full $A S F$ appears again for these issue-specific ones. However, the patterns change slightly with each issue. For example, while the most issue-specific ASFs fall substantially during the 112th House, the drop is quite small for Public Lands and Law and Order. During the 104th House, the issue-specific $A S F$ for the Civil Rights and Science issues drops substantially, while the $A S F$ for Public Lands increases substantially.

Jochim and Jones (2013) examine the dimensionality of legislative choice in the House (19652004), with focus on the relationship between party unity and issue dimensionality. They do so by scale roll-call votes separately in each PAP major topic code (W-NOMINATE) and use subjective inspection of scree-plots to determine dimensionality of each issue over time (Cattell 1966). There, they distinguish between distributive policy (e.g., trade, agriculture, public lands) and social policy (e.g., civil rights and social welfare). They expect distributive issues to be multidimensional because distributive policy is important for constituencies and hence for reelection. As a result, distributive policies are less subject to party pressure (Deering and Smith 1997). ${ }^{16}$ They find distributive issues such as science, trade, agriculture, public lands, and transportation to be multidimensional issues and find issues associated with intervention in the economy like housing and macroeconomics to be consistently uni-dimensional, owing to partisan conflict. ${ }^{17}$ We qualify these findings in a number of ways. First, while economic issues (e.g., housing, labor, macroeconomics) appear to be low-dimensional (high $A S F$ ) at one point in time (e.g., since the 110th), they are not uniquely low-dimensional compared to other issue-areas and certainly were not always 1-D issues. For example, in the 99th, these issues were among the most multidimensional. Second, we disagree with their finding that Environment and International Affairs were stable (or even

16 This is not to say our approach can directly estimate party influence in voting (Snyder Jr. and Groseclose 2000; Ansolabehere, Snyder Jr., and Stewart III 2001; McCarty et al. 2001; Cox and Poole 2002). Rather, extant methods of estimating party influence could be combined with our approach to provide a direct comparison of (differential) party effect in different domains of voting (e.g., Crook and Hibbing 1985; Jessee and Theriault 2014).

17 Mixed support for this argument can be seen in Gerrish and Blei (2012b) who find that energy and public lands (both distributive issues) show the most adjustment of member's preferences, but they also find economic issues such as appropriations and finance to be deviant as well. Jochim and Jones (2013) find economic issues to be consistently lowdimensional. 
increasing in dimensionality). We find both have decreased in dimensionality (at least until the 111th house)..$^{18}$ Lastly, while we agree that in general, dimensionality has been decreasing over time, but we find that the reasons for doing so may be multiple. Figure 2 show that while the overall level of extra-dimensionality at the chamber level may be similar over time, the reasons for this might be much different. For example, the 104th House is an example in which the dimensionality of issues varies enormously across issue-areas. Compare this to the 112th, which has roughly the same $A S F$. In the 112th, however, all issues have roughly similar issue-specific ASF.

\subsection{Comparison with Multidimensional Spatial Models}

Following our discussion from Section 3, one may wonder if our scaling technique is simply picking up extra dimensions estimated by traditional methods. That is, one may wonder if $A S F$ is simply capturing variance that could otherwise be explained by introducing additional dimensions in the model. To demonstrate that this is not always the case, we present in Figure 3, a comparison of the variance in the votes explained by the first dimension of a W-NOMINATE model (Poole and Rosenthal 2000) to our ASF metric. ${ }^{19}$ The curve connects the different Houses in time, and is meant to help visualize the joint evolution of both metrics. The trajectory in Figure 3 suggests that $A S F$ is indeed capturing something other than the lack of goodness-of-fit of a traditional 1-D spatial model, particularly in more recent years. Indeed, while both metrics have tended to increase over the last 35 years, they have not always done so simultaneously. The most striking example of divergence comes during Barack Obama's presidency, when the percentage of variability in the first dimension of W-NOMINATE remains high all along, but the ASF swings widely. Other examples include the transition from the 98th to 99th Houses, which shows an example of voting becoming more unidimensional according to W-NOMINATE but with a decreasing ASF, and the transitions from the 97th to 98th and from the 104th to 105th, where the $A S F$ remained approximately constant, but the percentage of variability in the first component of W-NOMINATE varied substantially. Likewise, in Reagan's era (e.g., 97th-100th Houses) ASF is consistently low, but the adequacy of (and evidence for) uni-dimensional space as estimated by NOMINATE varies considerably. Taken together, it is clear that the two metrics are capturing different aspects of political conflict.

\subsection{Legislator-Level Analysis}

One of the key contributions of our approach is its ability to provide micro-level information about the behavior of specific legislators. To this end, we introduce the individual staying frequency of a legislator $i$ :

$$
S F_{i}=\operatorname{Pr}\left(\zeta_{i, 1}=\zeta_{i, 2}=\cdots=\zeta_{i, K} \mid \text { Data }\right)
$$

This quantity is the posterior probability that legislator $i$ has consistent preferences across all domains of voting-that legislator $i$ is a stayer. ${ }^{20}$

18 The reason for these discrepancies likely lies in the "subsetting" of data and their method of inferring the dimensionality of an issue. Roberts et al. (2016) show that the level of aggregation of votes (committee, chamber, etc.) affects inference of dimensionality using roll-call votes. Further, by subsetting votes, estimates are necessarily less precise, increasing chance of reaching (some) incorrect conclusions (owning to increased sample variability).

19 Eigenvalues from W-NOMINATE are a tool to assess dimensionality of the underlying space. The eigenvalues provided by the W-NOMINATE analysis represent the amount of variability explained by each dimension. As expected, a large proportion of the total variability comes from the first. This proportion can be interpreted as a rough gauge of uni-dimensionality. Overall, we see that, for the U.S. House, a uni-dimensional model is commonly adequate, but the second dimension is not entirely without weight, a point made in Poole (2007).

20 An interesting theoretical question naturally arises: "why would a legislator have different revealed preferences in different domains of voting?" While a through treatment of this question is outside the scope of the current paper, in the context of the illustration, possible explanations might include: differential influence of factors affecting voting (Vandoren 1990); 


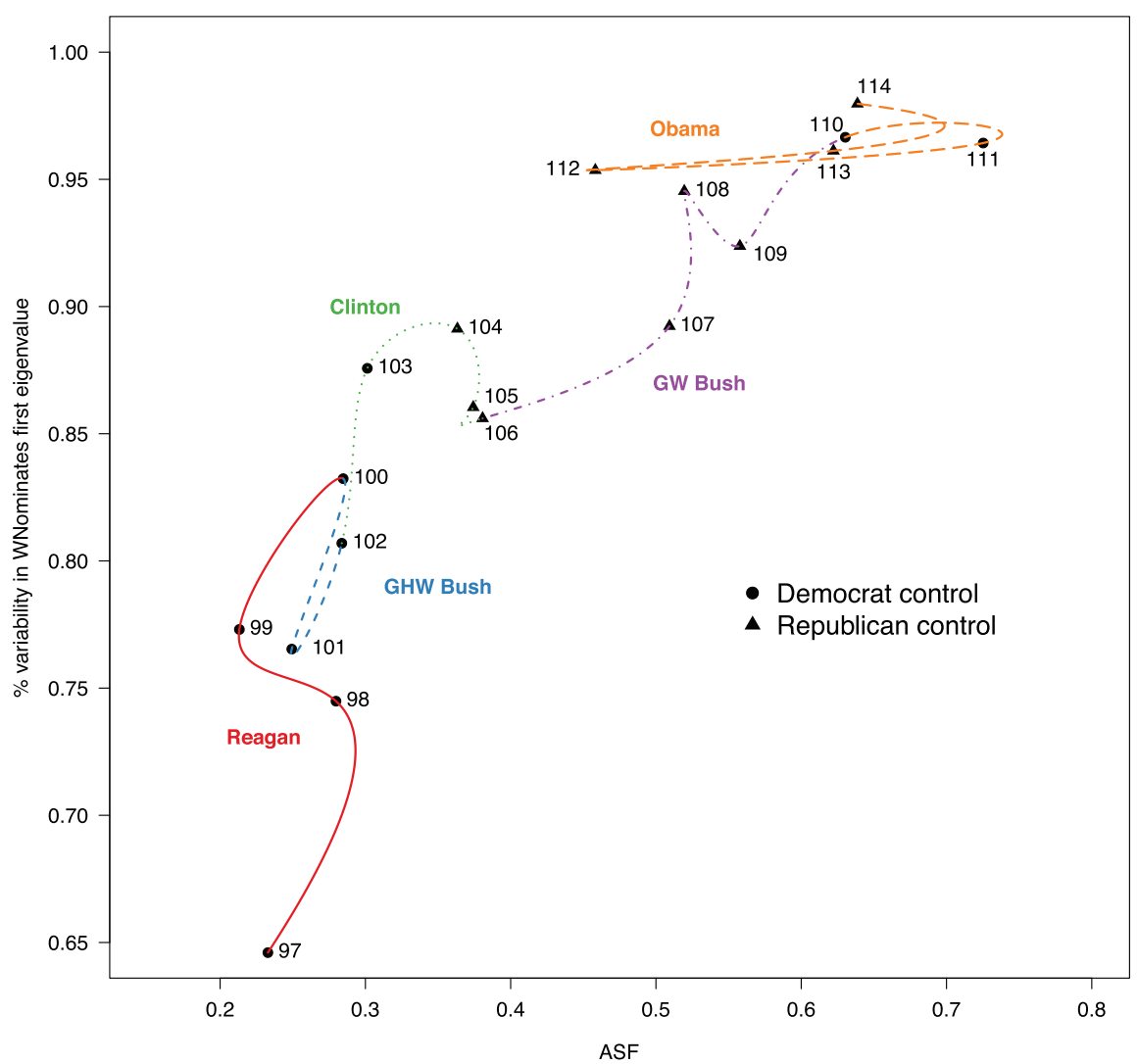

Figure 3. Ratio of the first eigenvalue to the sum of the first two eigenvalues from W-NOMINATE versus Average Staying Frequency (97th to 114th Houses). Shape represents majority party during the corresponding House (Democrats in circles, Republicans in triangles). Line type represents presidential eras.

We illustrate the legislator-level analysis using results from the 111th House. We focus on this particular House for this detailed analysis because it is the one with the smallest number of movers, and therefore, the easiest one to understand. Figure 4 shows the posterior median associated with the ideal point estimate rank of legislators arising from the standard, uni-dimensional model in (1) (along the $x$-axis) along with 95\% credible interval (in grey) for the 111th House. All legislators with a $S F$ lower than 0.5 are highlighted, although only some of the identities are included for legibility. Legislators that are not highlighted have a $S F$ higher than 0.5 and are considered full stayers. We can see that movers are distributed across the ranks, with most of the Democratic movers being relative centrists, and most of the Republican movers being to the right of the party. As a complement, we present in Figures 5 and 6 the identity of the 50 legislators with the lowest and highest $S F$ values, respectively. The legislators with the lowest $S F$ values are more or less equally distributed among the two parties. Furthermore, many of the mover's names are well known as individuals willing to buck their party and/or were cross-pressured by their constituency, for example, Young (R AK-1) who often appears as voting "deviant," and Rohrabacher (a Republican from California known for expressing positions contrary to the Republican party at the time, e.g., on immigration and Russia) and Berry (a Blue Dog Democrat from Arkansas). On the other hand, the vast majority ( 80\%) of the individuals with high $S F$ values belong to the Republican party.

Our model not only provides information about the identity of movers, but also about the nature of the issues in which they are movers, and the direction of preference changes. Similar

differential constituency interest/ attention to different policy areas (Clausen 1973; Peltzman 1984; Miler 2016). We note this as an avenue for future research in Section 6. 


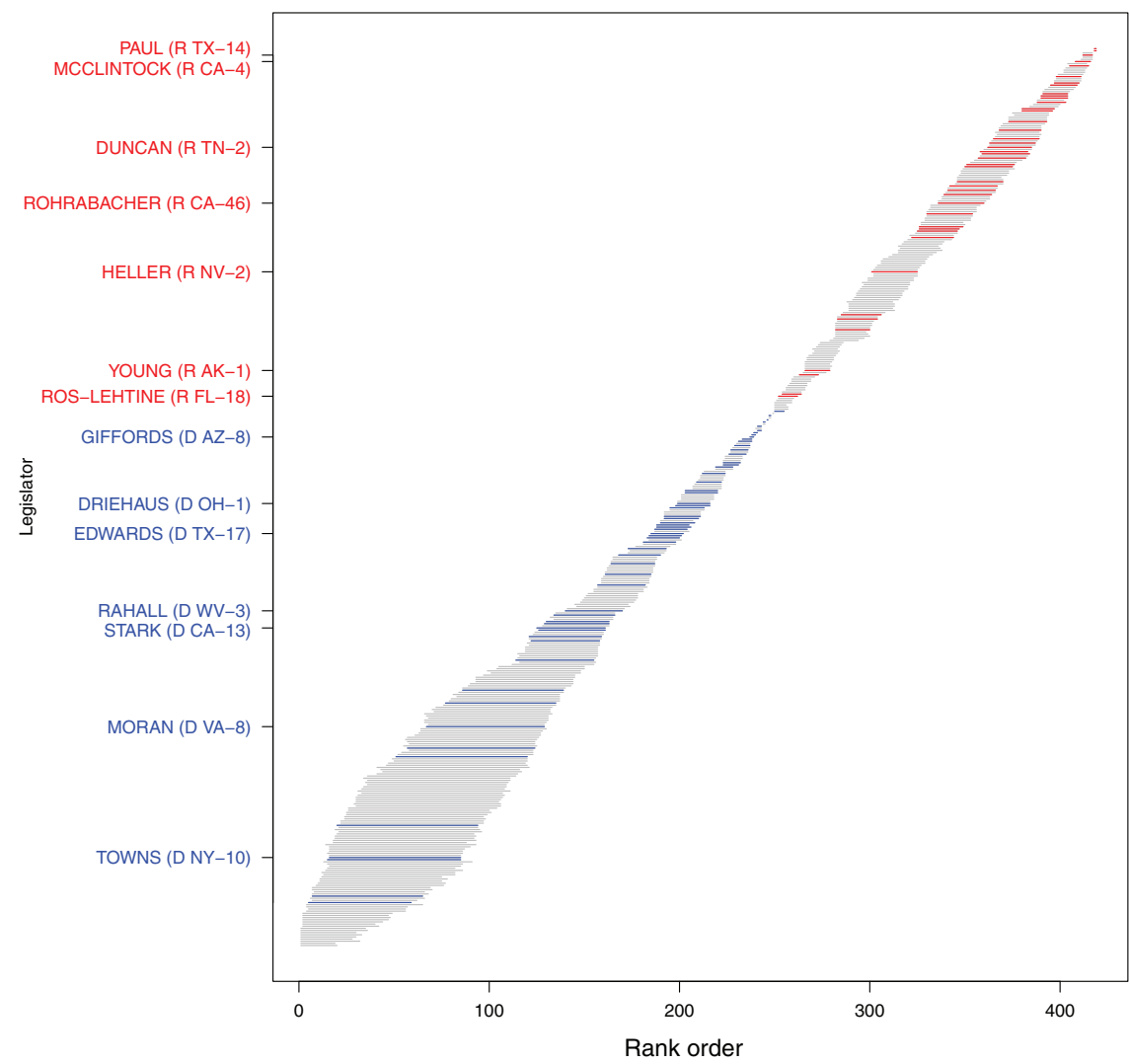

Figure 4. Ideal point estimates for 111th House from a uni-dimensional model with "movers" (i.e., legislators exhibiting a SF lower than 0.5) highlighted. There are 100 such legislators, only a subset of the names are included for readability.

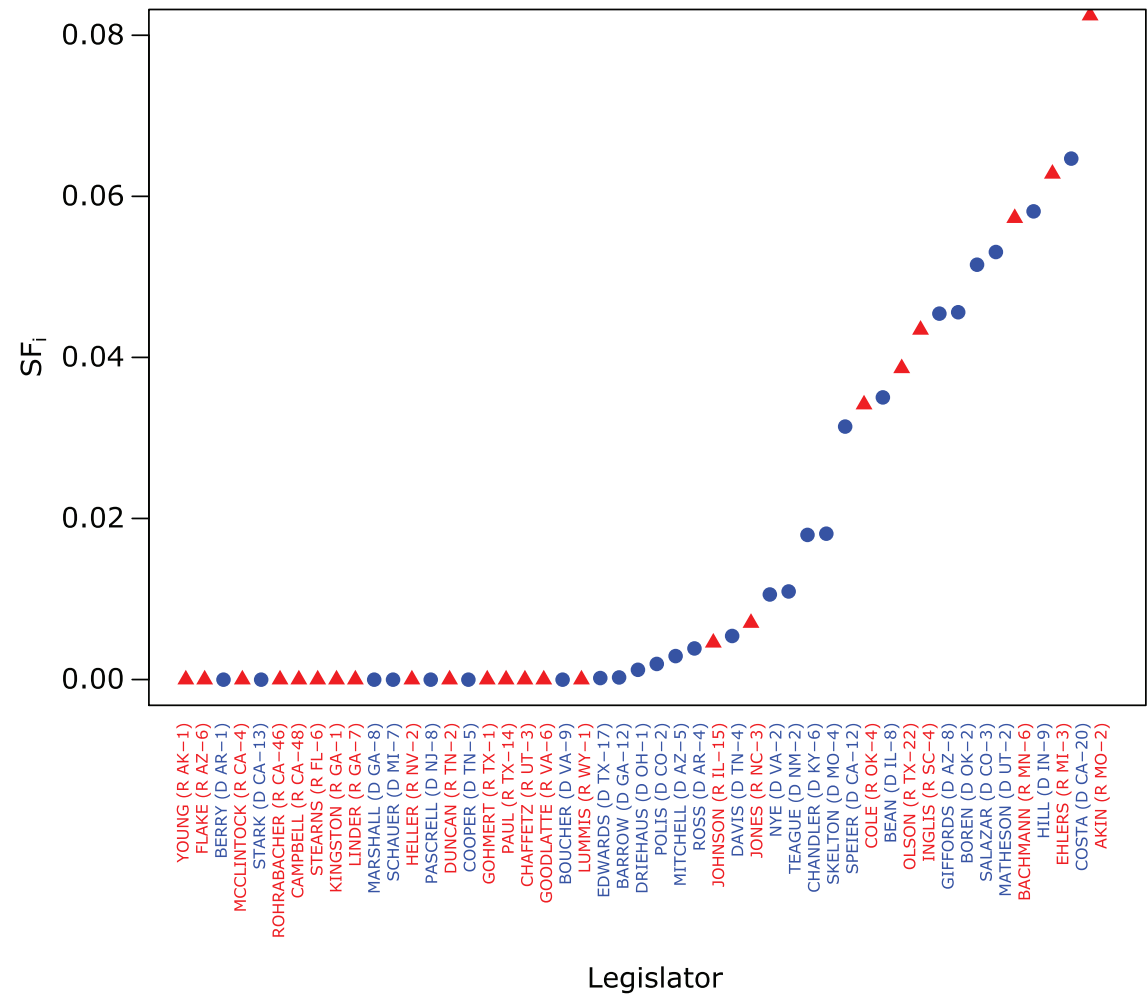

Figure 5. Legislators with the lowest $S F_{i}$ values during the 111th House. 


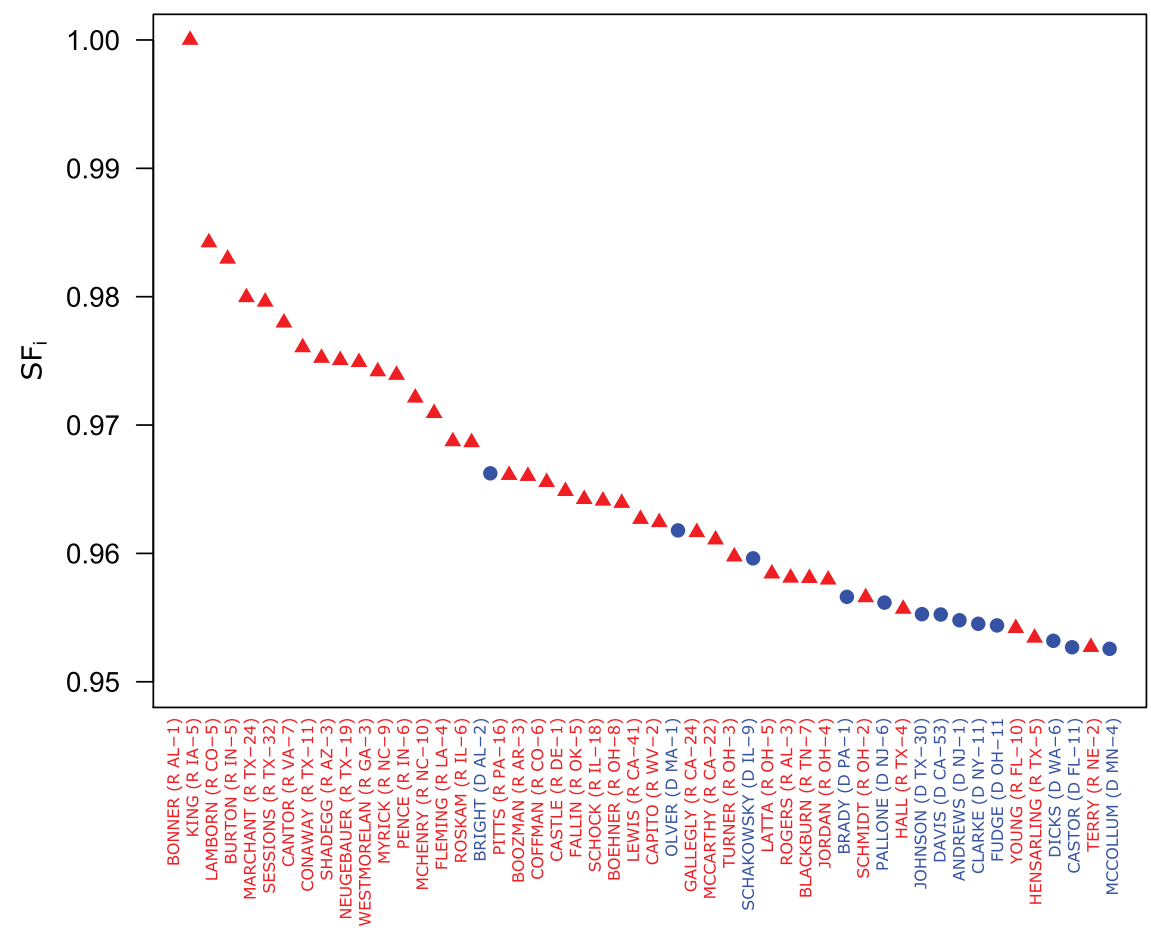

Legislator

Figure 6. Legislators with the highest $S F_{i}$ values during the 111th House.

to the procedure we used to define issue-specific $A S F s$, start by defining a member's basic ideal point as their ideal point in their base cluster, and say a member has deviant preferencespreferences different than their basic ideal point-on issues not in their base cluster. Figure 7 compares member's basic ideal point against the ideal points associated with four PAP issues for legislators in the 111th House (Banking and Finance, Civil Rights, Government Operations, and Macroeconomics). More specifically, these ternary plots show the probability that a member's ideal point in a specific issue is either left of, right of, or the same as their basic ideal point. An issue, $g$, is represented by three regions: the same revealed preference in that issue and a member's base cluster (no move, on the top); revealed preferences for issue $g$ more liberal than her base ideal point (leftward move, bottom left portion of a triangle); and revealed preference in issue $g$ more conservative than her base ideal point (rightward move, bottom right). Legislators in the top nomove region have a posterior probability that their revealed preference for issue $g$ is the same as their base ideal point greater than 0.5 . If a legislator's probability of moving is greater than 0.5 , then we classify the direction of the move depending on which is more likely.

Banking and finance is one example of an issue that exhibits clear and consistent partisan patterns of preference change. In particular, note that the vast majority of leftwards movers in this topic are Republicans, while the vast majority of rightward movers are Democrats. A similar pattern can be seen for Government Operations. We also observe issues on which members show no partisan or directional pattern of preference change, for example, macroeconomics.

We compare our illustration to Gerrish and Blei (2012b). While a direct comparison is not possible (as there voters have a main preference and issue-specific deviations), some of the insights provided by both models are similar. ${ }^{21}$ Gerrish and Blei (2012b) find votes on appropriations,

21 Arguably the main substantive finding in Gerrish and Blei (2012b) is that party polarization is greater on procedural votes than traditional ideal point estimates indicate. We consider procedural votes, votes at different stages of legislation and party polarization in a separate paper (Moser, Rodríguez, and Lofland 2019). 

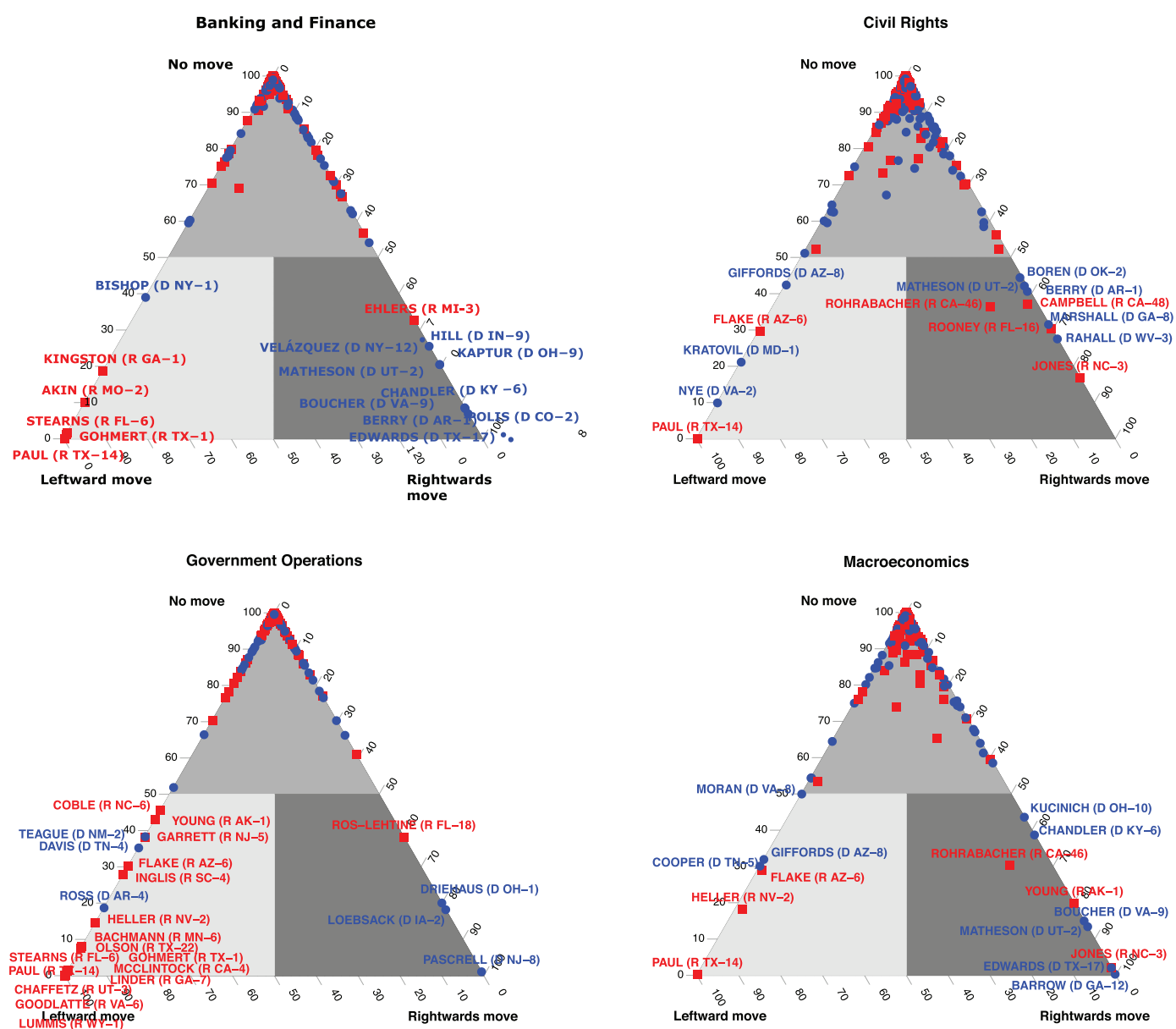

Figure 7. A selection of four issues in the 111th House, the name and direction of preference change.

finance, energy, and public lands to have the most movement (issues for which issue-specific preferences deviate the most from members' base preferences, roughly akin to our deviant issues). Likewise, they find that issues with the least amount of movement to be defence and military, education and foreign policy. Looking at the 111th House in particular, they find members preferences on Civil Rights to exhibit very little deviance from their base preference, which is somewhat different from our findings. We both, however, do find a considerable amount of movement/ deviance in the domain of Government Operations.

Figure 7 can also be used to track the behavior of specific legislators on different issues. We focus on two Representatives by way of example: Rep. Paul (R TX-14) and Rep. Young (R AK-1). ${ }^{22}$ Representative Paul (R TX-14) is estimated to be an extreme conservative (Figure 4) and a member with many multiple issue-specific ideal points (Figure 5). Representative Paul exhibits more liberal preferences (than his basic preference) in Banking, Civil Rights, Government Operations, and Macroeconomics (Figure 7). ${ }^{23}$ This is roughly consistent with his stated positions as a Libertarian. Conversely, Representative Young (R AK-1) is a moderate Republican (Figure 4) also with more than one ideal point (Figure 5). This member exhibits more conservative preferences on Microeconomics and more liberal preferences on Government Operations.

22 We focus on these two members primarily because they have been identified elsewhere as "extreme lawmakers" (Gerrish and Blei 2012b). We also identify them as such, but note several others as well, see Figure 4.

23 Gerrish and Blei (2012b) estimate Paul to have more liberal preferences on Civil Rights and more conservative preferences on Health and International Affairs. 


\subsection{Validation}

To provide some intuition and validation for the results discussed in the previous sections, the top panel of Figure 8 compares the (posterior median) ranks of voters in a particular vote-group (in this case, the ranking of member's revealed preferences in the domain of Government Operations during the 111th House) obtained by fitting a 1-D IDEAL model to that subset of votes alone, to the (posterior median) ranks obtained by fitting a 1-D IDEAL model to the whole set of votes. Intuitively, we expect members that our model estimates as movers would depart significantly from the diagonal. This approach mimics a procedure that has become common practice in the literature to identify changes in revealed preferences. Then, the bottom panel of Figure 8 compares the (posterior median) rank in the particular vote-group as estimated by our model against the same (posterior median) ranks obtained by fitting a 1-D IDEAL model to the whole set of votes.

Both graphs make it clear that there is a group of Republican legislators (most notably, Paul [R TX-14], Chaffetz [R UT-3], McClintock [R CA-4], Linder [T GA-7], Lummis [R WY], Goodlate [R VA-6], and Gohmert [R TX-1]) that demonstrate less conservative preferences in votes related to Government Operations than they do on other votes. ${ }^{24}$ However, there are also important differences between the two graphs. In particular, the graph that is based on our model is less noisy (bottom panel of Figure 8). This is because our joint model borrows information across voting domains to estimate the ideal points, leading to lower uncertainty.

In addition to providing initial validation our results, this simple example illustrates the challenges associated with using independent models for each issue as a tool to identify movers. In particular, it illustrates that the change in ranks for some of the legislators might be too small to be detected under the higher noise level associated with the relatively small samples involved with independent models. Figure 8 also highlights why attempting to address differences in voting preferences by visually comparing ranks across voting domains (rather than the underlying ideal points) can be quite misleading. For example, note that our model identifies as movers a number of relatively centrist legislators (such as Republicans Young [R TX-14] and Ros-Lehtine [R FL-27], and Democrats Davis [D TN-4], Driehaus [D OH-1], Ross [D AR-4], and Teagle [D NM-2]), whose ranks vary relatively little, particularly when compared against the large changes we observe for the group of Republican legislators discussed in the previous paragraph. These legislators would be particularly hard to identify as outliers in the top panel of Figure 8. Conversely, a number of Democrats near the median of the party exhibit large differences in the rankings across domains, but our model does not identify them as movers (as can be seen in the lower-left region of the graph in the top panel of Figure 8). A key issue to appreciate here is that the uncertainty associated with the estimates of ranks varies drastically. Generally speaking, the uncertainty associated with the rank of legislators that are close to the median voter of their own party tends to be much larger than the uncertainty associated with the ranks of centrist or radical legislators (Figure 4). Similarly, the uncertainty in the estimates of the ranks can vary substantially between parties (in the case of the 111th, the uncertainty associated with the ranks of Democrats is much larger than that of Republicans). As a consequence, changes in ranks for centrist, partisans and Republicans are easier to identify. Additionally, it is worth stressing that the ranks of the different legislators are not independent variables: If a legislator becomes more extreme, others will necessarily become less so. Hence, comparing ranks as outlined above (as opposed to comparing ideal points) cannot establish the source of the change. While the discussion above only addresses the issue of validity in one domain, and for one House, a similar pattern can be seen in the rest of our analyses.

24 A careful look into the history of these legislators suggests that this is not happening because they have moderate views in this topic. Instead, it is likely that this change in voting behavior is due to them casting protest votes. In other words, these legislators are likely voting against the Republican mainstream, rather than with the Democrats. This particular example serves to demonstrate the differences between revealed preferences (which is what spatial voting models are able to capture) and ideology. 

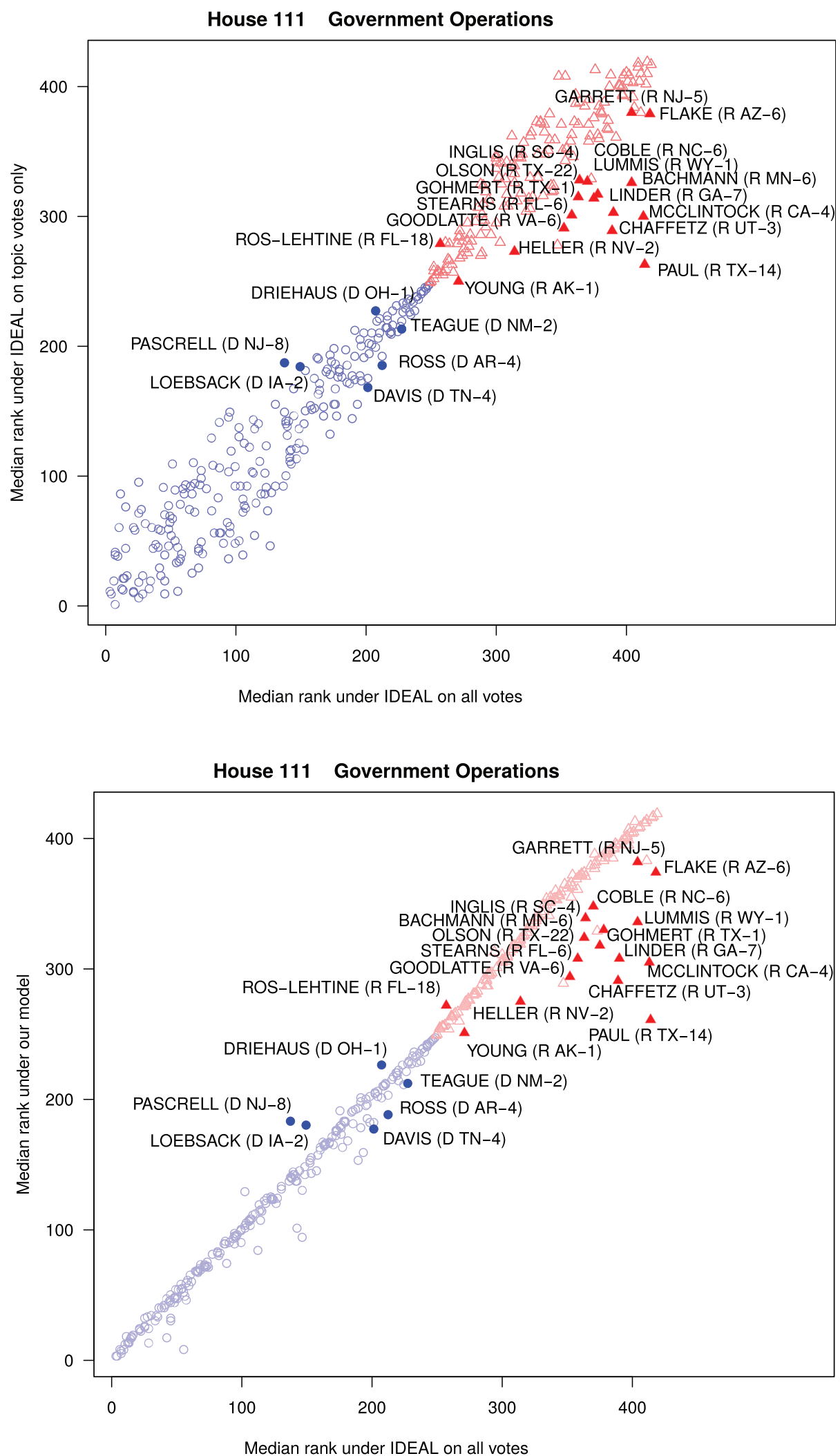

Figure 8. Comparison of posterior median ranks for legislators in the 111th House on Government Operations votes under various models (Democrats as circles; Republicans as triangles). Labled legislators correspond to those our model has identified as movers in this particular topic. 


\section{Discussion}

We developed a novel Bayesian model extending classical ideal point estimation methods to enable the joint estimation of voters' preferences across different domains of voting. The model places the resulting estimates of a member's ideal point on each of $K$ groups of votes on a common scale so that, for example, the revealed preferences of a member when voting on agriculture can be compared to their preferences on defense. The key innovation is to estimate, rather than assume, the identity of full stayers-voters with the same ideal point in all domains. This is achieved using a combination of mixture priors that ensure enough full stayers, along with classical identification techniques that anchors the scale.

We applied the model to the U.S. House of Representatives (97th to 114th) using PAP major topic codes to group votes. In this setting, we found that consistency of members' preferences (the posterior probability that a member exhibits the same revealed preferences in different issue areas) varies over time. At a chamber-level, the consistency is generally low until the 106th/107th House, when the chamber-level consistency increases dramatically (but with a notable exception in the 112th, see Figure 1). The reason for low preference consistency across issues also differs: many legislators may have deviant preferences on a small set of issues (as in the 104th); or there may be many issues that legislators show deviant preferences on (e.g., 112th, see Figure 2). Using the PAP grouping of votes, we explored how preferences differ across issues.

\subsection{Other Potential Applications}

We end with some possible applications of the model presented here in different avenues of study.

- Our estimation technique results in a common measurement of the revealed preferences of voters in different domains (this measurement might or might not be one-dimensional). This allows for a direct comparison across domains, to compliment indirect comparisons of behavior across domains. The commonality of the measurement (e.g., scale) allows for comparison of individuals (e.g., "does member $X$ vote more liberally on issue $A$ than on issue $B$ ?") and of groups (e.g., "are parties more polarized/unified/dispersed on procedural votes than amendments or final passage?"). The latter question is important for both questions of party polarization (Roberts and Smith 2003; Theriault 2008; Duff and Rohde 2012) and legislative organization (Cox and McCubbins 1993, 2005).

- Our approach allows us to test sharp hypotheses. This is a new feature in the literature. For example, we may now formally test the claim "[t]he presence of departing members [in lame-duck sessions] created a legislative environment marked by increased ideological and participatory shirking..." (Nokken 2014, p. 440) by comparing ideal points in regular and lame-duck sessions estimated on a common scale..$^{25}$

- The methodology developed here is a kind of "partial pooling" model for roll-call votes/IRT scaling, resulting in more precise estimates (a feature analogous to the "information borrowing" found in multilevel models).

- Related, this approach provides a principled and unified approach to the "granularity" issue in the analysis of roll-call data (Crespin and Rohde 2010; Roberts et al. 2016). The issue in that subliterature is the level of aggregation used in roll-call analysis. ${ }^{26}$ It has been noted in the literature that the level of aggregation of roll-call votes used in ideal point estimation matters for the resulting dimensionality of the latent space (Crespin and Rohde 2010). ${ }^{27}$ The

25 There is nothing wrong with the analysis in Nokken (2014), which compares the frequency of different "types" of votes in regular and lame-duck sessions along with passage rates in the two sessions. But ideally, the research would compare the distribution of ideal points (and features thereof, e.g., variation) between different votes types. This is precisely what our method allows for, among other features.

26 "... when we compare more narrowly defined issue areas, it might no longer be plausible to explain voting with only one or two dimensions" (Crespin and Rohde 2010, p.981).

27 Scholars have argued-and presented both empirical and theoretical support-that the finer the grouping of votes, the more likely multidimensional latent spaces are found (Harding 2008; Roberts et al. 2016). Conversely, when all votes in a session are used in scaling, 1 or 2 dimensions swamp other aspects of political conflict (Poole 2007; Aldrich et al. 2014). 
model presented here offers an alternative approach to the question of level-of-votes used. By scaling all votes together, but allowing votes to be distinguished by type (e.g., issue), we borrow information across vote types while maintaining a common scale, and allowing the dimensionality of the issue space to depend on the legislator.

- There are applications to the dimensionality of voting literature (Poole et al. 1991; Wilcox and Clausen 1991; Potoski and Talbert 2000; Roberts et al. 2016). It is well-known that even though the overall structure of voting may be summarized via a unidimensional model, the underlying votes may not be (Crespin and Rohde 2010; Dougherty et al. 2014; Roberts et al. 2016). Less is known about the dimensionality of individual voter's preferences. Our approach allows for estimation of legislator-specific dimensionality.

These methodological enhancements have several possible applications and allow for new questions to be asked. We list some here.

- First, we note that some theories of legislative committees posit (require, actually) that committee members are preference outliers relative to the floor (Denzau and Mackay 1983; Shepsle and Weingast 1987; Weingast and Marshall 1988). Some empirical tests of these claims rely on third parties' assessments of members' preferences (e.g., ADA scores, Krehbiel 1990). By grouping committee member's votes by context (committee, floor), our approach allows for a direct comparison of voting in committee versus voting on the floor.

In that setting, a kind of scarcity is present: all committee members (can) vote both in their committee and on the floor; but noncommittee members cannot vote in committee, only on the floor. Nonetheless, our approach allows for a direct comparison of committee members' revealed preference with those when voting in committee to the floor (e.g., the median member's ideal point on the floor). This is seemingly relevant for the committeeoutlier debate in the House (Snyder Jr. 1992a, 1992b; Groseclose 1994a, 1994b; Brown et al. 1997).

Related, one could use the common scale arising from our estimator to study committeefloor voting and gate-keeping (Snyder Jr. 1992b) by comparing the position of ideal points revealed in committee to those revealed on the floor.

- Recently, we have used the common scale produced by our method to study the dynamics of polarization in the House (Moser et al. 2019). There, we use the comparability of ideal points to study the dynamics of polarization in different procedural domains of voting. We find that voting on amendments contributes to increasing polarization in the modern House to a greater degree than previously found (Theriault 2008; Pump 2010; Jessee and Theriault 2014).

- Since estimation results in a partition of vote types (issues) for each member, one may perform a clustering analysis of issues. On which issues do members have the same revealed preferences (which issues appear in member's clusters together)? Exploring these issueassociations over time may be of use for the study of issue-evolution (Clausen and Cheney 1970; Carmines and Stimson 1986, 1990; Wilcox and Clausen 1991; Baumgartner et al. 2009; Lee and Schutte 2017). Indeed, our approach could be used to integrate the a priori approach with the a postiori approach. Our model is hence useful for engaging with some of the fundamental research questions of the policy-dimensions approach: "... identify[ing] individual members and groups of members that deviate from a consistent ideological position. We can then explain these deviations in terms of substance: the limitations of the unidimensional view are revealed by the meaningfulness of the deviations from it. Such deviations are meaningful in terms of party, region, constituency, and philosophy of government" (Wilcox and Clausen 1991, p. 404).

- One could, in principle, examine the representative-constituency connection by, for example, regressing changes in revealed preferences in a specific policy (e.g., agriculture) on constituency characteristics, among others. We also note that most studies of issue-specific preferences (e.g., Clausen 1973; Wilcox and Clausen 1991; Anderson 2012) rely on extrachamber factors, such as interests group ratings. Our approach could potentially contribute 
to that literature by providing estimates of issue-specific preferences that are comparable across issues.

- As we mentioned in Section 3, the approach taken here is useful for exploring the difference between the basic space and the issue space (Poole 2007; Benoit and Laver 2012) by incorporating both inductive and deductive approaches to the interpretation of dimensions. Related, issue-specific preferences are a matter of interest for a number of scholars, for example, Clausen and Wilcox (1987), Clausen (1973), Sulfaro (2000), Anderson (2012), Jochim and Jones (2013). The estimator developed here has potential usefulness to such scholars as it permits estimation of preference on a specific issue that is comparable to preferences on other issues.

- Lastly, we note that our approach permits new questions to be studied: when members exhibit different revealed preferences in different domains, why do they do so? For example, past scholarship has identified preferences over agricultural policy to be substantially different then general ideology (Mayhew 1966; Hurwitz, Moiles, and Rohde 2001). We are now in a position to attempt to explain such differences quantitatively (our method gives a way to construct a quantitative "deviance" (of preference) on for example, agriculture. One could use this as a dependent variable in a regression on, for example, constituency attributes or other factors used in the representation literature (Miller and Stokes 1963; McCrone and Kuklinski 1979; Peltzman 1984; Poole and Romer 1993; Brennan and Hamlin 1999; Clinton 2006; Miler 2010; Lapinski 2013; Sulkin, Testa, and Usry 2015; Miler 2016).

\section{Appendix A. Derivation of the Prior on the Concentration Parameter $\phi$}

Our goal is to select a prior for $\phi$ such that the induced prior on $\theta=\frac{\Gamma(K) \Gamma(\phi+1)}{\Gamma(K+\phi)}$, the probability that any legislator is a stayer, follows a uniform distribution on the unit interval. The density of such prior must satisfy $p(\phi)=\left|\frac{\mathrm{d} \theta}{\mathrm{d} \phi}\right|$, and has support on the $[0, \infty)$ interval. Now,

$$
\begin{aligned}
\left|\frac{\mathrm{d} \theta}{\mathrm{d} \phi}\right| & =\Gamma(K)\left|\frac{\Gamma^{\prime}(\phi+1)}{\Gamma(K+1)}-\frac{\Gamma(\phi+1) \Gamma^{\prime}(\phi+K)}{[\Gamma(\phi+K)]^{2}}\right| \\
& =\Gamma(K) \frac{\Gamma(\phi+1)}{\Gamma(\phi+K)}\left|\frac{\Gamma^{\prime}(\phi+1)}{\Gamma(\phi+1)}-\frac{\Gamma^{\prime}(\phi+K)}{\Gamma(\phi+K)}\right| \\
& =\Gamma(K) \frac{\Gamma(\phi+1)}{\Gamma(\phi+K)}\{\psi(\phi+K)-\psi(\phi+1)\} \\
& =\Gamma(K) \frac{\Gamma(\phi+1)}{\Gamma(\phi+K)}\left\{\sum_{k=0}^{K-2} \frac{1}{\phi+k+1}\right\},
\end{aligned}
$$

where $\psi(\cdot)=\frac{\Gamma^{\prime}(\cdot)}{\Gamma(\cdot)}$ denotes the digamma function. Note that, for $K=2$, the prior reduces to $p(\phi)=$ $(\phi+1)^{-2}$, a (shifted) Pareto distribution. Figure A1 shows the shape of this prior distribution for two different values of $K$. Note that the prior tends to concentrate more around zero for higher values of $K$. It is also worthwhile noting that, while this is a proper distribution, it is heavy tailed. Indeed, since $\Gamma(\phi+1) / \Gamma(\phi+K)=1 / Q_{K-1}(\phi)$, where $Q_{K-1}$ is a polynomial of degree $K-1$, this prior has only $K-2$ finite moments. 


\section{Appendix B. Number of Votes by Vote-Type, U.S. House of Representatives}

Table B1. Frequency of PAP major topic recorded votes by House. Source: Policy Agendas Project.

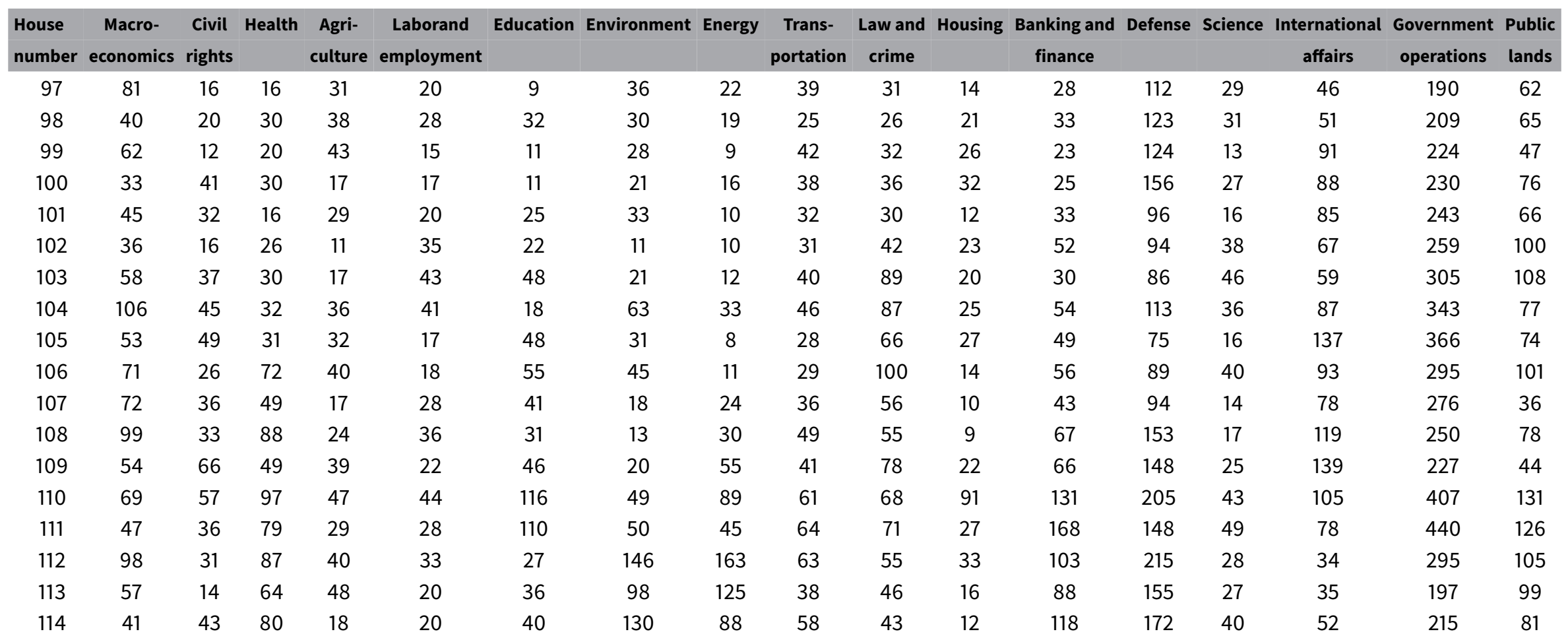




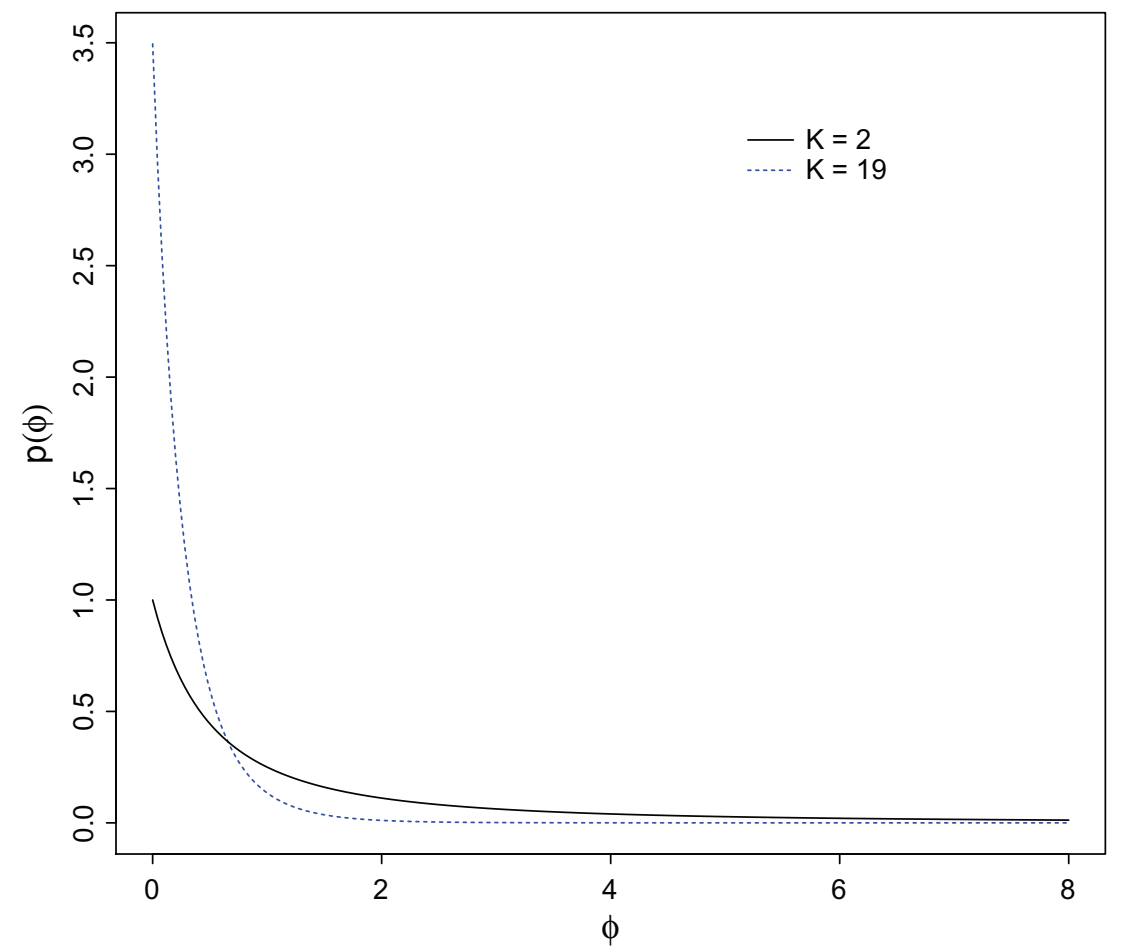

Figure A1. Prior distribution for the concentration parameter $\phi$ for various values of $K$.

\section{Acknowledgments}

An earlier version of this work was presented at the 2017 Midwest Political Science Association Annual Conference under the title "Comparing Revealed Preferences Across Multiple Types of Motions in the 83rd to 112th, U.S. House of Representatives. This work has benefited from helpful comments from Marc Ratkovic, as well as from three anonymous referees. Abel Rodriguez was partially supported by award NSF-DMS 1738053 and 1740850.

\section{Data Availability Statement}

Replication code for this article has been published in Code Ocean, a computational reproducibility platform that enables users to run the code and can be viewed interactively at https://doi.org/10.24433/CO.5298256.v1 (Moser, Rodriguez, and Lofland 2020a). A preservation copy of the same code and data can also be accessed via Dataverse at https://doi.org/10.7910/DVN/ STH14F (Moser, Rodriguez, and Lofland 2020b).

\section{References}

Adler, E. S., and J. Wilkerson. 2017. Congressional Bills Project [data file and codebook].

Albert, J. H., and S. Chib. 1993. "Bayesian Analysis of Binary and Polychotomous Response." American Statistical Association 88(422):669-679.

Aldrich, J. H., J. M. Montgomery, and D. B. Sparks. 2014. "Polarization and Ideology: Partisan Sources of Low Dimensionality in Scaled Roll Call Analyses." Political Analysis 22(2):435-456.

Anderson, S. E. 2012. "Policy Domain-Specific Ideology: When Interest Group Scores Offer More Insight." Politics \& Policy 40(6):1186-1202.

Ansolabehere, S., J. M. SnyderJr., and C. Stewart III. 2001. "The Effects of Party and Preferences on Congressional Roll-Call Voting.” Legislative Studies Quarterly 26(4):533-572.

Antoniak, C. 1974. "Mixtures of Dirichlet Processes with Applications to Bayesian Nonparametric Problems." Annals of Statistics 2:1152-1174.

Armstrong, H., C. K. Carter, K. F. K. Wong, and R. Kohn. 2009. "Bayesian Covariance Matrix Estimation Using a Mixture of Decomposable Graphical Models." Statistics and Computing 19(3):303-316.

Asmussen, N., and J. Jo. 2016. "Anchors Away: A New Approach for Estimating Ideal Points Comparable Across Time and Chambers." Political Analysis 24(2):172-188. 
Bafumi, J., A. Gelman, D. K. Park, and N. Kaplan. 2005 "Practical Issues in Implementing and Understanding Bayesian Ideal Point Estimation.” Political Analysis 13:171-87.

Bakker, R., S. Jolly, J. Polk, and K. Poole. 2014. "The European Common Space: Extending the Use of Anchoring Vignettes." The Journal of Politics 76(4):1089-1101.

Baumgartner, F. R., C. Breunig, C. Green-Pedersen, B. D. Jones, P. B. Mortensen, M. Nuytemans, and S. Walgrave. 2009. "Punctuated Equilibrium in Comparative Perspective." American Journal of Political Science 53(3):603-620.

Baumgartner, F. R., and B. D. Jones. 1993. Agendas and Instability in American Politics. Chicago: University of Chicago Press.

Benoit, K., and M. Laver. 2012. "The Dimensionality of Political Space: Epistemological and Methodological Considerations." European Union Politics 13(2):194-218.

Binder, S. A. 1999. "The Dynamics of Legislative Gridlock, 1947-96." American Political Science Review 93(3):519-533.

Brennan, G., and A. Hamlin. 1999. "On Political Representation.” British Journal of Political Science 29(1):109-127.

Brown, R. D., J. M. Davis, L. M. Overby, C. E. S. Jr, and D. R. Holian. 1997. "The Dynamics of Committee Outliers: Evidence from the House of Representatives, 1951-90." The Journal of Legislative Studies 3(2):70-88.

Cameron, C. M. 2000. Veto Bargaining: Presidents and the Politics of Negative Power. Cambridge: Cambridge University Press.

Carmines, E. G., and J. A. Stimson. 1986. "On the Structure and Sequence of Issue Evolution." American Political Science Review 80(3):901-920.

Carmines, E. G., and J. A. Stimson. 1990. Issue Evolution: Race and the Transformation of American Politics. Princeton, NJ: Princeton University Press.

Carroll, R., J. B. Lewis, J. Lo, K. T. Poole, and H. Rosenthal. 2009. "Comparing NOMINATE and IDEAL: Points of Difference and Monte Carlo Tests." Legislative Studies Quarterly 34(4):555-591.

Carroll, R., J. B. Lewis, J. Lo, K. T. Poole, and H. Rosenthal. 2013. "The Structure of Utility in Spatial Models of Voting." American Journal of Political Science 57(4):1008-1028.

Cattell, R. B. 1966. "The Scree Test for the Number of Factors." Multivariate Behavioral Research 1(2) 245-276.

Celeux, G. 1998. “Bayesian Inference for Mixture: The Label Switching Problem.” In COMPSTAT: Proceedings in Computational Statistics, edited by R. Payne and P. Green, 227-232. New York: Springer.

Clarke, K. A., and D. M. Primo. 2012. A Model Discipline: Political Science and the Logic of Representations. Oxford: Oxford University Press.

Clausen, A. 1973. How Congressmen Decide: A Policy Focus. New York: St. Martin's Press.

Clausen, A. R., and R. B. Cheney. 1970. "A Comparative Analysis of Senate-House Voting on Economic and Welfare Policy, 1953-1964.” American Political Science Review 64(1):138-152.

Clausen, A. R., and C. Wilcox. 1987. "Policy Partisanship in Legislative Leadership Recruitment and Behavior." Legislative Studies Quarterly 1(2):243-263.

Clinton, J., S. Jackman, and D. Rivers. 2004. "The Statistical Analysis of Roll Call Data." American Political Science Review 98(2):355-370.

Clinton, J. D. 2006. "Representation in Congress: Constituents and Roll Calls in the 106th House." The Journal of Politics 68(2):397-409.

Clinton, J. D., and S. Jackman. 2011. "To Simulate or NOMINATE?" Legislative Studies Quarterly 34(4):593-621.

Clinton, J., S. Jackman, and D. Rivers. 2004. "The Statistical Analysis of Roll Call Data.” American Political Science Review 98(2):355-370.

Cox, G. W., and M. D. McCubbins. 1993. Legislative Leviathan: Party Government in the House. California Series on Social Choice and Political Economy. Berkeley: University of California Press.

Cox, G. W., and M. D. McCubbins. 2005. Setting the Agenda: Responsible Party Government in the US House of Representatives. Cambridge: Cambridge University Press.

Cox, G. W., and K. T. Poole. 2002. "On Measuring Partisanship in Roll-Call Voting: The U.S. House of Representatives, 1877-1999.” American Journal of Political Science 46(3):477.

Crespin, M. H., and D. W. Rohde. 2010. "Dimensions, Issues, and Bills: Appropriations Voting on the House Floor." The Journal of Politics 72(4):976-989.

Crook, S. B., and J. R. Hibbing. 1985. "Congressional Reform and Party Discipline: The Effects of Changes in the Seniority System on Party Loyalty in the US House of Representatives." British Journal of Political Science 15(2):207-226.

Davis, O. A., M. J. Hinich, and P. C. Ordeshook. 1970. "An Expository Development of a Mathematical Model of the Electoral Process." American Political Science Review 64(2):426-448.

De Vries, C. E., and G. Marks. 2012. "The Struggle over Dimensionality: A Note on Theory and Empirics." European Union Politics 13(2):185-193.

Deering, C. J., and S. S. Smith. 1997. Committees in Congress. New York: CQ Press. 
Denzau, A. T., and R. J. Mackay. 1983. "Gatekeeping and Monopoly Power of Committees: An Analysis of Sincere and Sophisticated Behavior." American Journal of Political Science 27(4):740-761.

Dougherty, K. L., M. S. Lynch, and A. J. Madonna. 2014. "Partisan Agenda Control and the Dimensionality of Congress." American Politics Research 42(2):600-627.

Dowding, K., A. Hindmoor, and A. Martin. 2013. "The Policy Agendas Project: Reflections on Theory." Chicago, IL: Social Science Research Network.

Dowding, K., A. Hindmoor, and A. Martin. 2016. "The Comparative Policy Agendas Project: Theory, Measurement and Findings." Journal of Public Policy 36(1):3-25.

Duclos, J.-Y., J. Esteban, and D. Ray. 2004. “Polarization: Concepts, Measurement, Estimation.” Econometrica 72(6):1737-1772.

Duff, J. F., and D.W. Rohde. 2012. "Rules to Live by: Agenda Control and the Partisan Use of Special Rules in the House." Congress \& the Presidency 39(1):28-50.

Egar, W. T. 2016. "Tarnishing Opponents, Polarizing Congress: The House Minority Party and the Construction of the Roll-Call Record." Legislative Studies Quarterly 41(4):935-964.

Enelow, J. M., and M. J. Hinich. 1984. The Spatial Theory of Voting: An Introduction. Cambridge: Cambridge University Press.

Esteban, J.-M., and D. Ray. 1994. "On the Measurement of Polarization." Econometrica 62(4):819-851.

Gerrish, S. M., and D. M. Blei. 2010. "The Ideal Point Topic Model: Predicting Legislative Roll Calls from Text." In Proceedings of the Computational Social Science and the Wisdom of Crowds Workshop. Neural Information Processing Symposium, Citeseer.

Gerrish, S. M., and D. M. Blei. 2012a. "How They Vote: Issue-Adjusted Models of Legislative Behavior." In Advances in Neural Information Processing Systems 25, edited by F. Pereira, C. J. C. Burges, L. Bottou, and K. Q. Weinberger, 2753-2761. New York: Curran Associates, Inc.

Gerrish, S. M., and D. M. Blei. 2012b. “The Issue-Adjusted Ideal Point Model.” Preprint, arXiv:1209.6004.

Geweke, J. 1992. "Evaluating the accuracy of sampling-based approaches to the calculations of posterior moments." In Bayesian Statistics 4, edited by J. M. Bernardo, J. Berger, A. P. Dawid, and A. F. M. Smith, 641-649. Oxford: Oxford University Press.

Gopalan, R., and D. A. Berry. 1998. "Bayesian Multiple Comparisons Using Dirichlet Process Priors." Journal of the American Statistical Association 93(443):1130-1139.

Groseclose, T. 1994a. "Testing Committee Composition Hypotheses for the U.S. Congress." The Journal of Politics 56(2):440-458.

Groseclose, T. 1994b. "The Committee Outlier Debate: A Review and a Reexamination of Some of the Evidence.” Public Choice 80(3):265-273.

Harding, M. C. 2008. "Explaining the Single Factor Bias of Arbitrage Pricing Models in Finite Samples." Economics Letters 99(1):85-88.

Hurwitz, M. S., R. J. Moiles, and D. W. Rohde. 2001. "Distributive and Partisan Issues in Agriculture Policy in the 104th House." American Political Science Review 95(4):923-937.

Jackman, S. 2001. "Multidimensional Analysis of Roll Call Data via Bayesian Simulation: Identification, Estimation, Inference, and Model Checking." Political Analysis 9(3):227-241.

Jessee, S. A. 2012. Ideology and Spatial Voting in American Elections. Cambridge: Cambridge University Press.

Jessee, S. A., and S. M. Theriault. 2014. "The Two Faces of Congressional Roll-Call Voting." Party Politics 20(6):836-848.

Jochim, A. E., and B. D. Jones. 2013. "Issue Politics in a Polarized Congress.” Political Research Quarterly 66(2):352-369.

Jones, B. D., and F. R. Baumgartner. 2005. The Politics of Attention: How Government Prioritizes Problems. Chicago: University of Chicago Press.

Koford, K. 1989. "Dimensions in Congressional Voting." American Political Science Review 83(3): 949-962.

Krehbiel, K. 1990. "Are Congressional Committees Composed of Preference Outliers?" American Political Science Review 84(1):149-163.

Krehbiel, K. 1998. Pivotal Politics: A Theory of U.S. Lawmaking. Chicago: University of Chicago Press.

Krehbiel, K., A. Meirowitz, and J. Woon. 2005. "Testing Theories of Lawmaking." In Social Choice and Strategic Decisions, edited by D. Austen-Smith and J. Duggan. New York: Springer.

Lapinski, J. S. 2013. The Substance of Representation: Congress, American Political Development, and Lawmaking. Vol. 133. Princeton, NJ: Princeton University Press.

Lau, J. W., and P. J. Green. 2007. "Bayesian Model-Based Clustering Procedures.” Journal of Computational and Graphical Statistics 16(3):526-558.

Lauderdale, B. E., and T. S. Clark. 2014. "Scaling Politically Meaningful Dimensions Using Texts and Votes." American Journal of Political Science 58(3):754-771.

Lee, D. J., and R. A. Schutte. 2017. "Elite-Level Issue Dynamics: Assessing Perspectives on Party Issue Change." Party Politics 23(3):205-219.

Liu, C., D. B. Rubin, and Y. N. Wu. 1998. "Parameter Expansion to Accelerate EM: The PX-EM Algorithm." Biometrika 85(4):755-770. 
Lofland, C. L., A. Rodríguez, and S. Moser. 2017. "Assessing Differences in Legislators' Revealed Preferences: A Case Study on the 107th U.S. Senate." Annals of Applied Statistics 11(1):456-479.

Londregan, J. 1999. "Estimating Legislators' Preferred Points." Political Analysis 8(1):35-56.

Lord, F. 1952. A Theory of Test Scores. Psychometric Monograph, Income Red. New York: Psychometric Society. Martin, A. D., and K. M. Quinn. 2002. "Dynamic Ideal Point Estimation via Markov Chain Monte Carlo for the U.S. Supreme Court, 1953-1999.” Political Analysis 10(2):134-153.

Mayhew, D. R. 1966. Party Loyalty among Congressmen: The Difference between Democrats and Republicans, 1947-1962. Cambridge, MA: Harvard University Press.

McCarty, N. M., and K. T. Poole. 1995. "Veto Power and Legislation: An Empirical Analysis of Executive and Legislative Bargaining from 1961 to 1986." Journal of Law, Economics and Organization 11:282-312.

McCarty, N. M., K. T. Poole, and H. Rosenthal. 1997. Income Redistribution and the Realignment of American Politics. Washington: AEI Press.

McCarty, N. M., K. T. Poole, and H. Rosenthal. 2001. "The Hunt for Party Discipline in Congress." American Political Science Review 95(3):673-687.

McCrone, D. J., and J. H. Kuklinski. 1979. "The Delegate Theory of Representation." American Journal of Political Science 23(2):278-300.

Miler, K. 2016. "Legislative Responsiveness to Constituency Change." American Politics Research 44(5):816-843.

Miler, K. C. 2010. Constituency Representation in Congress: The View from Capitol Hill. Cambridge: Cambridge University Press.

Miller, W. E., and D. E. Stokes. 1963. "Constituency Influence in Congress." American Political Science Review 57(1):45-56.

Moffett, K. W. 2012. "Parties and Procedural Choice in the House Rules Committee." Congress \& the Presidency 39(1):1-27.

Moser, S., A. Rodriguez, and C. Lofland. 2020a. "Multiple Ideal Points: Revealed Preferences in Different Domains." Code Ocean V1:29. https://codeocean.com/capsule/0712643/tree/v1.

Moser, S., A. Rodriguez, and C. Lofland. 2020b. "Replication Data for: Multiple Ideal Points: Revealed Preferences in Different Domains." https://doi.org/10.7910/DVN/STH14F, Harvard Dataverse, V1, UNF:6:3I1dnh+PIOnvpGL0O3yXSA== [fileUNF].

Moser, S., A. Rodríguez, and C. L. Lofland. 2019. "Polarization and Amendments: The Overlooked Role of Amendments in Explaining Increased Polarization." Working Paper.

Neal, R. M. 2000. "Markov chain sampling methods for Dirichlet process mixture models." Journal of Computational and Graphical Statistics 9(2):249-265.

Nokken, T. P. 2014. "Comparing Agenda Content and Roll-Call Behaviour in Regular and Lame-Duck Sessions of the House of Representatives, 1879-2010." The Journal of Legislative Studies 20(4):430-450.

Peltzman, S. 1984. "Constituent Interest and Congressional Voting." The Journal of Law \& Economics 27(1):181-210.

Pitman, J. 1996. "Some Developments of the Blackwell-MacQueen Urn Scheme." In Statistics, Probability, and Game Theory: Papers in Honor of David Blackwell, edited by D. Blackwell, T. S. Ferguson, L. S. Shapley, and J. B. MacQueen, 245-267. Lecture Notes-Monograph Series. New York: Institute of Mathematical Statistics.

Policy Agendas Project . 2017. Roll-Call Votes.

Poole, K. T. 2007. "Changing Minds? Not in Congress!” Public Choice 131(3-4):435-451.

Poole, K. T., and T. Romer. 1993. "Ideology, 'Shirking', and Representation." In The Next Twenty-Five Years of Public Choice, edited by C. Rowley, F. Schneider, and R. D. Tollison, 185-196. New York: Springer.

Poole, K. T., and H. Rosenthal. 1985. "A Spatial Model for Legislative Roll Call Analysis." American Journal of Political Science 29(2):357-384.

Poole, K. T., and H. Rosenthal. 1987. "Analysis of Congressional Coalition Patterns: A Unidimensional Spatial Model." Legislative Studies Quarterly 12(1):55-75.

Poole, K. T., and H. Rosenthal. 1991. "Patterns of Congressional Voting." American Journal of Political Science 35(1):228-278.

Poole, K. T., and H. Rosenthal. 2000. Congress: A Political-Economic History of Roll Call Voting. Oxford: Oxford University Press.

Poole, K. T., and H. L. Rosenthal. 2011. Ideology and Congress. New York: Transaction Publishers.

Poole, K. T., H. Rosenthal, and K. Koford. 1991. "On Dimensionalizing Roll Call Votes in the US Congress." American Political Science Review 85(3):955-976.

Potoski, M., and J. Talbert. 2000. "The Dimensional Structure of Policy Outputs: Distributive Policy and Roll Call Voting." Political Research Quarterly 53(4):695-710.

Pump, B. 2010. Beyond Roll Calls: Institutional Change and Partisanship in the US House of Representatives. SSRN Scholarly Paper ID 1580563. Rochester, NY: Social Science Research Network.

Rasch, G. 1960. Probabilistic Models for Some Intelligence and Attainment Tests. Studies in Mathematical Psychology. Copenhagen: Danish Institute for Educational Research.

Rivers, D. 2003. Identification of multidimensional item-response models. Technical report. Department of Political Science, Stanford University. 
Robert, C. P., and G. Casella. 2005. Monte Carlo Statistical Methods. 2nd edn. New York: Springer.

Roberts, J. M. 2010. "The Development of Special Orders and Special Rules in the U.S. House, 1881-1937." Legislative Studies Quarterly 35(3):307-336.

Roberts, J. M., and S. S. Smith. 2003. "Procedural Contexts, Party Strategy, and Conditional Party Voting in the U.S. House of Representatives, 1971-2000." American Journal of Political Science 47(2):305-317.

Roberts, J. M., S. S. Smith, and S. R. Haptonstahl. 2016. "The Dimensionality of Congressional Voting Reconsidered." American Politics Research 44(5):794-815.

Scott, J. G., and J. O. Berger. 2006. "An Exploration of Aspects of Bayesian Multiple Testing." Journal of Statistical Planning and Inference 136(7):2144-2162.

Scott, J. G., and J. O. Berger. 2010. "Bayes and Empirical-Bayes Multiplicity Adjustment in the Variable-Selection Problem.” The Annals of Statistics 38(5):2587-2619.

Shepsle, K. A., and B. R. Weingast. 1987. "The Institutional Foundations of Committee Power." American Political Science Review 81(1):85-104.

Shor, B., C. Berry, and N. McCarty. 2010. "A Bridge to Somewhere: Mapping State and Congressional Ideology on a Cross-Institutional Common Space." Legislative Studies Quarterly 35(3):417-448.

Shor, B., and N. M. McCarty. 2011. "The Ideological Mapping of American Legislatures." American Political Science Review 10503:530-551.

SnyderJr. , J. M., and T. Groseclose. 2000. "Estimating Party Influence in Congressional Roll-Call Voting." American Journal of Political Science 44(2):193-211.

Snyder, J. M. , Jr. 1992a. "Committee Power, Structure-Induced Equilibria, and Roll Call Votes." American Journal of Political Science 36(1):1-30.

Snyder, J. M. , Jr. 1992b. "Gatekeeping or Not, Sample Selection in the Roll Call Agenda Matters." American Journal of Political Science 36(1):36-39.

Stephens, M. 2000. "Dealing with Label Switching in Mixture Models." Journal of the Royal Statistical Society: Series B (Statistical Methodology) 62(4):795-809.

Stokes, D. E. 1963. "Spatial Models of Party Competition.” American Political Science Review 57(2):368-377.

Sulfaro, V. A. 2000. "Sources of Structure in Congressional Behavior: The Influence of Ideology on Foreign and Domestic Policy Votes." Southeastern Political Review 28(1):77-110.

Sulkin, T., P. Testa, and K. Usry. 2015. "What Gets Rewarded? Legislative Activity and Constituency Approval." Political Research Quarterly 68(4):690-702.

Talbert, J. C., and M. Potoski. 2002. "Setting the Legislative Agenda: The Dimensional Structure of Bill Cosponsoring and Floor Voting." The Journal of Politics 64(3):864-891.

Theriault, S. M. 2008. Party Polarization in Congress. Cambridge: Cambridge University Press.

Treier, S. 2011. "Comparing Ideal Points across Institutions and Time.” American Politics Research 39(5):804-831.

Tsebelis, G. 1995. "Decision Making in Political Systems: Veto Players in Presidentialism, Parliamentarism, Multicameralism and Multipartyism." British Journal of Political Science 25(3):289.

Vandoren, P. M. 1990. “Can We Learn the Causes of Congressional Decisions from Roll-Call Data?” Legislative Studies Quarterly 15(3):311.

Weingast, B. R., and W. J. Marshall. 1988. "The Industrial Organization of Congress; or, Why Legislatures, like Firms, Are Not Organized as Markets.” Journal of Political Economy 96(1):132-163.

Wilcox, C., and A. Clausen. 1991. "The Dimensionality of Roll-Call Voting Reconsidered." Legislative Studies Quarterly 16(3):393-406. 